\title{
Filament Capturing with the Multimaterial Moment-of-Fluid Method *
}

\author{
Matthew Jemison ${ }^{\dagger}$ \\ Department of Applied \& Computational Mathematics \\ Florida State University \\ Mark Sussman \\ Department of Applied \& Computational Mathematics \\ Florida State University \\ Mikhail Shashkov $\ddagger$ \\ X-Computational Physics Division \\ Los Alamos National Laboratory
}

January 2, 2015

\begin{abstract}
A novel method for capturing two-dimensional, thin, under-resolved material configurations, known as "filaments," is presented in the context of interface reconstruction. This technique uses a partitioning
\end{abstract}

${ }^{*}$ Work supported in part by the National Science Foundation under contract DMS 1016381.

${ }^{\dagger}$ This work of M. Jemison was performed under the auspices of the U.S. Department of Energy's NNSA as part of Los Alamos National Laboratory's Computational Physics Student Summer Workshop organized by Scott Runnels. Los Alamos National Laboratory is operated by Los Alamos National Security, LLC, under contract number DE-AC5206NA25396.

†This work of M. Shashkov was performed under the auspices of the National Nuclear Security Administration of the US Department of Energy at Los Alamos National Laboratory under Contract No. DE-AC52-06NA25396 and supported by the DOE Advanced Simulation and Computing (ASC) program. M. Shashkov also acknowledges the partial support of the DOE Office of Science ASCR Program. 
procedure to detect disconnected regions of material in the advective preimage of a cell (indicative of a filament) and makes use of the existing functionality of the Multimaterial Moment-of-Fluid interface reconstruction method to accurately capture the under-resolved feature, while exactly conserving volume. An algorithm for Adaptive Mesh Refinement in the presence of filaments is developed so that refinement is introduced only near the tips of filaments and where the Moment-Of-Fluid reconstruction error is still large. Comparison to the standard Moment-of-Fluid method is made. It is demonstrated that using filament capturing at a given resolution yields gains in accuracy comparable to introducing an additional level of mesh refinement at significantly lower cost.

\section{Introduction}

In the field of interface reconstruction, the problem of a material configuration under-resolved by the mesh is often overcome by increasing mesh resolution. Regions of high-curvature can be detected and captured by using Adaptive Mesh Refinement [21, 2, 12], hybrid particle level set method[6], or by introducing oriented cell subzones[7]. However, in the case of thin structures, it may be impractical to increase mesh resolution to fully resolve the structure.

Thin structures in material configuration can lead to reconstruction errors, resulting in erroneous material breakup [4, 9, 8]. Piecewiselinear interface reconstruction techniques that use non-local information to determine interface orientation, such as Volume of Fluid (VOF) and Young's method, exhibit "numerical surface tension," an artifact of the interface reconstruction method that can induce erroneous breakup.

The method of [9] examines the material occupying a shared node with respect to neighboring cells and detects discrepancies indicating interfacial breakup. In cells where a discrepancy is detected, interface smoothing is applied to repair topology after advection. Similarly, the method of [3] uses volume fraction data and material ID information at vertices to determine interface topology, generate a discretized material interface from a look-up table, and last perform a smooth, volume-accurate material interface reconstruction. The techniques in [9] and [3] do not ultimately remove the problems of filaments thinner than grid cells. 
Increasing the sophistication of the Volume-of-Fluid reconstruction algorithm, either by piecewise parabolic reconstruction[16] or by the advecting normals technique[15], improves the performance for simulating surface tension driven free surface flows, but do not address the problems when a filament is thinner than the mesh size.

High order level set methods[11, 13, 22] naturally represent filaments even when a filament is thinner than a grid cell. Unfortunately numerical methods for solving the level set advection equation do not obey a geometric conservation law. There is no guarantee of volume conservation when representing interfaces with the level set method. Also, the accuracy of the level set method is dependent on both the accuracy of the numerical scheme and dependent on the flow velocity away from the interface. Large gradients in the level set function can develop[23] in a flow with non-uniform velocity, thereby defeating the benefit of high order level set advection schemes. In order to prevent the build-up of large gradients in the level set function, the level set function is often reinitialized to be a distance function[23, 14]. Unfortunately, whereby reinitialization schemes prevent the buildup of large gradients in the level set function where the interface is smooth, the level set gradient in underresolved filamentary regions will be increased, defeating the purpose of a high order method.

In this paper, we use existing functionality of the Multimaterial Moment-of-Fluid method to reconstruct filamentary interfaces underresolved by the mesh in a unique way. Rather than introduce tracer particles, or otherwise increase the resolution in filamentary regions, we introduce a fictitious fluid to capture under-resolved regions. We introduce a new "conglomeration algorithm" that determines whether a computational cell's advective preimage contains a filament, and therefore a single material needs to be broken up into two materials. By introducing the fictitious material, we pose the reconstruction step as three materials separated by two interfaces, rather than two materials separated by a single interface. This method automatically preserves volume exactly since the volume preserving property of the multimaterial Moment-Of-Fluid recontruction algorithm[5] carries over to our "filament capturing" algorithm. In contrast to adaptive mesh approaches, our method is asymptotically constant in spatial complexity as the width of the filament goes to zero.

Remarks:

- The polygonal area mapping method[25] has similarities with our approach in that all polygonal regions in the advective preimage 
are investigated in determining the complexity of the polygonal structure to be stored in a computational cell. Our method is different from [25] in how we compress the preimage information in order to prevent an exponential growth of polygonal information per cell. Also our method has the capability to capture the creation of filaments for all advected materials instead of just one.

- If one explicitly tracked a deforming boundary using connected markers (see e.g. [24]), then filamentary regions are trivially preserved at the expense of one having to introduce extra logic in order to modify the connectivity of markers when opposite sides of a material become "close." Recent grid based front tracking methods[19, 20,17] automatically reconnect markers when interfaces are about to merge, but the criteria for interface merging is implicitly dependent on the underlying grid. The resolution of the underlying grid determines the thickness of resolvable filaments. In our approach, the adjacency condition (see Section 4.2) for analyzing material volumes in the advective preimage determines connectivity; in contrast to front tracking approaches, the parameters for determining whether interfaces touch depends on floating point precision, instead of a grid dependent or a user dependent parameter.

The paper is organized as follows. First the multi-material Momentof-Fluid interface reconstruction and advection algorithm is presented. Then, a conglomeration procedure is described which is used to detect disconnected regions of the same material from the advective preimage of a cell, indentifying the formation of a filament. The Moment-ofFluid interface reconstruction problem is then formulated as a three material problem by relabeling the disconnected regions as two different materials. The algorithm for Adaptive Mesh Refinement is developed in the context of the conglomeration method to accurately capture the tips of filaments without refining the mesh more than necessary. Finally, numerical results are presented, demonstrating the increased performance in accuracy and runtime of the filament-capturing technique. 


\section{Moment-of-Fluid Interface Reconstruc- tion}

In a computational cell $\Omega_{i}$, the reference volume fraction, $F_{i}^{m}$, and the reference first order moment, $\boldsymbol{x}_{i}^{m}$, are given for each material $m$, $m=1, \ldots, M$. For the scenario $M=2$, materials are separated by an interface $\Gamma_{i}$, which is represented with a piecewise-linear interface reconstruction in each cell. The Moment-of-Fluid Method (MOF) reconstruction uses the reference volume fractions (zeroth order moment) and centroids (2.2) of a material region (i.e. the first moment) in determining the orientation of the interface [5]. The reconstructed interface in a cell is the piecewise-linear interface (2.3) that exactly captures the reference volume fraction (2.1) and minimizes error in the centroid (2.2) for a material $m$. Centroid error can be interpreted as curvature in the interface, which can be used as a condition for mesh refinement in AMR algorithms [2].

$$
\begin{gathered}
\hat{F}_{i}^{m}=\frac{\int_{\Omega_{i}^{m}} d \Omega}{\int_{\Omega_{i}} d \Omega} \\
\boldsymbol{x}_{i}^{m}=\frac{\int_{\Omega_{i}^{m}} \boldsymbol{x} \cdot d \Omega}{\int_{\Omega_{i}} d \Omega} \\
\Gamma(\boldsymbol{x}, b)=\hat{n} \cdot \boldsymbol{x}+b
\end{gathered}
$$

Using the Cell-Integrated Semi-Lagrangian advection method in Section 3, materials are transported into a target cell. As materials in the departure region (advective preimage) are advected, they undergo deformation based on the characteristic mapping that translates material from the departure region to the target cell. Reference volume fractions $\left(F_{r e f}^{m}\right)$ and reference centroids $\left(\boldsymbol{x}_{r e f}^{m}\right)$ are then calculated in the cell of interest. An interface $\Gamma(\hat{n}, b)$ cutting the cell will yield an actual volume fraction $\left(F_{a c t}^{m}\right)$ and actual centroid $\left(\boldsymbol{x}_{a c t}^{m}\right)$, which are functions of the parameters $\hat{n}$ and $b$. The optimal interface will satisfy $(2.4)$

$$
\left\{\begin{array}{l}
\left|F_{a c t}^{m}(\hat{n}, b)-F_{r e f}^{m}\right|=0 \\
\underset{\hat{n}, b}{\arg \min }\left|\boldsymbol{x}_{a c t}^{m}(\hat{n}, b)-\boldsymbol{x}_{r e f}^{m}\right|
\end{array}\right.
$$

To limit numerical round-off errors, the reference volume fractions are normalized to add to one, and materials with a volume fraction 
below a cut-off tolerance are truncated to zero, as in (2.6) for the volume fraction $F^{m}$ of an arbitrary material $m$. The tolerance was taken to be $\epsilon=10^{-8}$ by experiment.

$$
\begin{gathered}
F^{m}=\frac{\hat{F}^{m}}{\sum_{i=1}^{M} \hat{F}^{i}} \\
F^{m}= \begin{cases}0, & F^{m} \leq \epsilon \\
F^{m}, & \epsilon<F^{m}<1-\epsilon \\
1, & F^{m} \geq 1-\epsilon\end{cases}
\end{gathered}
$$

For the scenario when the number of materials $M$ in cell $\Omega_{i}$ is greater than 2, the Moment Of Fluid reconstruction procedure is generalized as follows:

1. Initialize the uncaptured space in a cell to be $\Omega_{u}=\Omega_{i}$ where $\Omega_{i}$ represents cell $i$. The centroid of $\Omega_{u}$ is denoted as $\boldsymbol{x}_{u}$. Tag all materials as "not defined."

2. If the number of undefined materials with $F_{m}>0$ is greater or equal to two, then

$$
m^{n e x t}=\operatorname{argmax}_{m, F_{i}^{m}>0, m \text { not defined }}\left|\boldsymbol{x}_{i}^{m}-\boldsymbol{x}_{u}\right| .
$$

3. Find the slope $\hat{n}$ and intercept $b$ that minimizes the centroid error with the constraint that $\left|F_{a c t}^{m^{\text {next }}}(\hat{n}, b)-F_{\text {ref }}^{m^{\text {next }}}\right|=0(2.4) . \Omega_{m^{\text {next }}}$ is defined as:

$$
\Omega_{m^{n e x t}}=\Omega_{u} \cap\left\{\boldsymbol{x} \mid \hat{n} \cdot\left(\boldsymbol{x}-\boldsymbol{x}_{i}\right)+b \geq 0\right\}
$$

The Gauss-Newton method is used to solve the optimization problem defined by (2.4) [10]. Tag material $m^{\text {next }}$ as "defined."

4. Update $\Omega_{u}$ and go back to step 2 :

$$
\Omega_{u}=\Omega_{u} \cap \Omega_{m^{\text {next }}}^{\text {Complement }}
$$

In contrast to earlier implementations of the Moment-of-Fluid Interface Reconstruction method that used a Gauss-Green discretization to compute volumes and moments of polygonal regions [1], our implementation makes use of triangulation to compute reference volumes and reference centroids. Each material region is sub-divided into triangles (Fig. 1). The intersection of two triangulated regions is 
expressed as the union of triangles. A lookup table is utilized to efficiently cut a triangle with a linear interface and triangulate the cut region. Volume of the cut region is then computed as the sum of the volumes of the constituent triangles. Since all polygonal regions are decomposed into triangles, it is easy to compute moments. For a given a triangle $T$, the centroid $\boldsymbol{x}_{T}$ is the average of its vertices $\boldsymbol{x}_{j}(2.10)$.

$$
\boldsymbol{x}_{T}=\frac{\int_{T} \boldsymbol{x} \cdot d \boldsymbol{x}}{\int_{T} d \boldsymbol{x}}=\frac{1}{3} \sum_{j=1}^{3} \boldsymbol{x}_{j}
$$

If a polygonal region $P(2.11)$ is written as the union of $N$ triangles $T_{i}$, each with volume $V_{i}$ and centroid $\boldsymbol{x}_{T_{i}}$,

$$
P=\bigcup_{i=1}^{N} T_{i}
$$

the volume $V_{P}$ of the region $P$ is equal to the sum of the volumes of $T_{i}$. The centroid $\boldsymbol{x}_{P}$ of $P$ is the volume-weighted sum of the centroids (2.13).

$$
\begin{gathered}
V_{P}=\sum_{i=1}^{N} V_{i} \\
\boldsymbol{x}_{P}=\frac{\int_{P} \boldsymbol{x} \cdot d \boldsymbol{x}}{\int_{P} d \boldsymbol{x}}=\frac{\sum_{i=1}^{N} V_{i} \boldsymbol{x}_{T_{i}}}{V_{P}}
\end{gathered}
$$

We remark that our Moment-Of-Fluid multimaterial reconstruction procedure allows the intersection of interfacial cuts within a computational cell in contrast to the more restrictive "onion skin" strategy often employed together with Volume-Of-Fluid techniques [18]. If a computational cell contains $P$ materials with $F_{P}>0$, then there will be $P-1$ linear cuts in the cell, some cuts potentially intersecting previous cuts. Our algorithm can reconstruct interface configurations where 3 materials meet at a single point, albeit one of the angles separating 2 of the materials must be 90 degrees. 


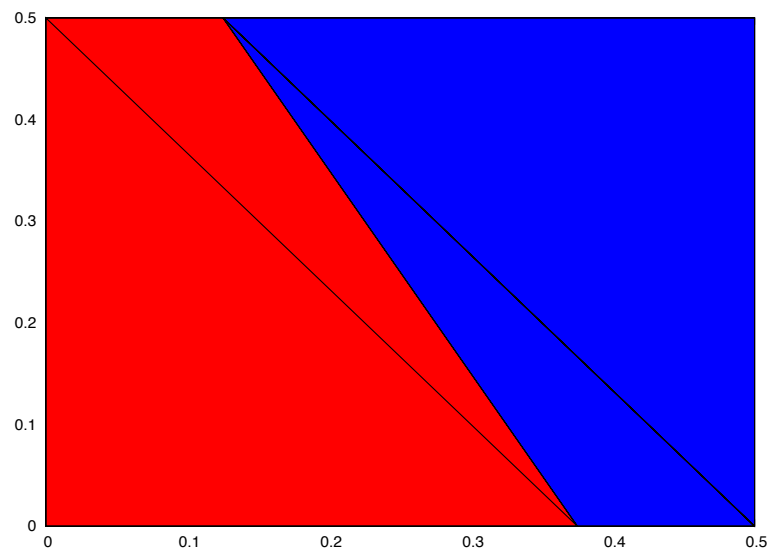

Figure 1: Rectangular cell $\Omega=[0,1 / 2] \times[0,1 / 2]$ is cut by linear interface, $\Gamma=-2 x-y+3 / 4$. Cut regions are triangulated, allowing for easy volume and moment calculation.

\section{Multimaterial Advection}

An unsplit Cell-Integrated Semi-Lagrangian (CISL) method is used for material transport, with characteristics computed using second-order Runge-Kutta. Velocity is defined at cell nodes, and the characteristic ODE (3.1) is solved at each vertex to define a set of back-traced vertices.

Each computational cell $\Omega_{i}$ is partitioned into two target triangles. For each target triangle $T_{t}$, the corresponding "departure region" ("advective preimage") is the region bounded by the 3 back-traced vertices of the target triangle $T_{t}$ (Fig. 2). This is the material which will depart its current location and move into $T_{t}$.

$$
\dot{\boldsymbol{x}}=-\boldsymbol{v}(t, \boldsymbol{x})
$$

The mapping from a departure triangle $T_{d}$ to a target triangle $T_{t}$ is defined as follows:

1. Let $\boldsymbol{x}_{t, 1}, \boldsymbol{x}_{t, 2}, \boldsymbol{x}_{t, 3}$ be the vertices of $T_{t}$ and $\boldsymbol{x}_{d, 1}, \boldsymbol{x}_{d, 2}, \boldsymbol{x}_{d, 3}$ the vertices of $T_{d}$.

2. Define a linear mapping $L \boldsymbol{x}$ that satisfies:

$$
L: T_{d} \rightarrow T_{t}
$$


In other words,

$$
L \boldsymbol{x}_{d} \equiv A\left(\boldsymbol{x}_{d}-\boldsymbol{x}_{d, 1}\right)+\boldsymbol{x}_{t, 1}
$$

The first row of $A$ satisfies,

$$
\left(\begin{array}{cc}
x_{d, 2}-x_{d, 1} & y_{d, 2}-y_{d, 1} \\
x_{d, 3}-x_{d, 1} & y_{d, 3}-y_{d, 1}
\end{array}\right)\left(\begin{array}{c}
a_{11} \\
a_{12}
\end{array}\right)=\left(\begin{array}{c}
x_{t, 2}-x_{t, 1} \\
x_{t, 3}-x_{t, 1}
\end{array}\right)
$$

The second row of $A$ satisfies,

$$
\left(\begin{array}{cc}
x_{d, 2}-x_{d, 1} & y_{d, 2}-y_{d, 1} \\
x_{d, 3}-x_{d, 1} & y_{d, 3}-y_{d, 1}
\end{array}\right)\left(\begin{array}{c}
a_{21} \\
a_{22}
\end{array}\right)=\left(\begin{array}{l}
y_{t, 2}-y_{t, 1} \\
y_{t, 3}-y_{t, 1}
\end{array}\right)
$$

The departure triangle $T_{d}$ is intersected with the underlying mesh, which is also triangularized, each underlying mesh cell $\Omega_{j}$ broken up into two triangles, which produces many triangles $T_{d, \text { intersect }}$ which are mapped according to $L$ (3.2) into the target region. Since $L$ is a linear mapping, the image of $T_{d, \text { intersect }}$ in $T_{t}$ will be a triangle too. The image of the linear interface(s) $\Gamma$ under the action of $L$ will still be a linear interface but with a modified slope. If $\Gamma$ is defined as,

$$
\Gamma=\left\{\boldsymbol{x} \mid \boldsymbol{n} \cdot\left(\boldsymbol{x}-\boldsymbol{x}_{j}\right)+b=0\right\},
$$

then $L \Gamma$ is defined as,

$$
\begin{array}{r}
L \Gamma=\left\{L \boldsymbol{x} \mid \boldsymbol{n} \cdot\left(\boldsymbol{x}-\boldsymbol{x}_{j}\right)+b=0\right\}= \\
\left\{\boldsymbol{x} \mid \boldsymbol{n}_{\text {image }} \cdot\left(\boldsymbol{x}-L \boldsymbol{x}_{j}\right)+b=0\right\},
\end{array}
$$

where,

$$
\boldsymbol{n}_{\text {image }}=\left(A^{-1}\right)^{T} \boldsymbol{n} .
$$

Remarks:

- The solvability of the system 3.9 is guaranteed under the condition that at no point during the continuous mapping from the departure triangle $T_{d}$ to the target triangle $T_{t}$ does the triangular region collapse to zero volume. If the nodes of the triangulated sub-region become co-linear during the Lagrangian transport phase, the mapping becomes singular. Solvability can be guaranteed under suitable CFL constraints $(C F L<1 / 2)$, or by directly solving for the time at which a triangular sub-region will collapse to zero volume. 

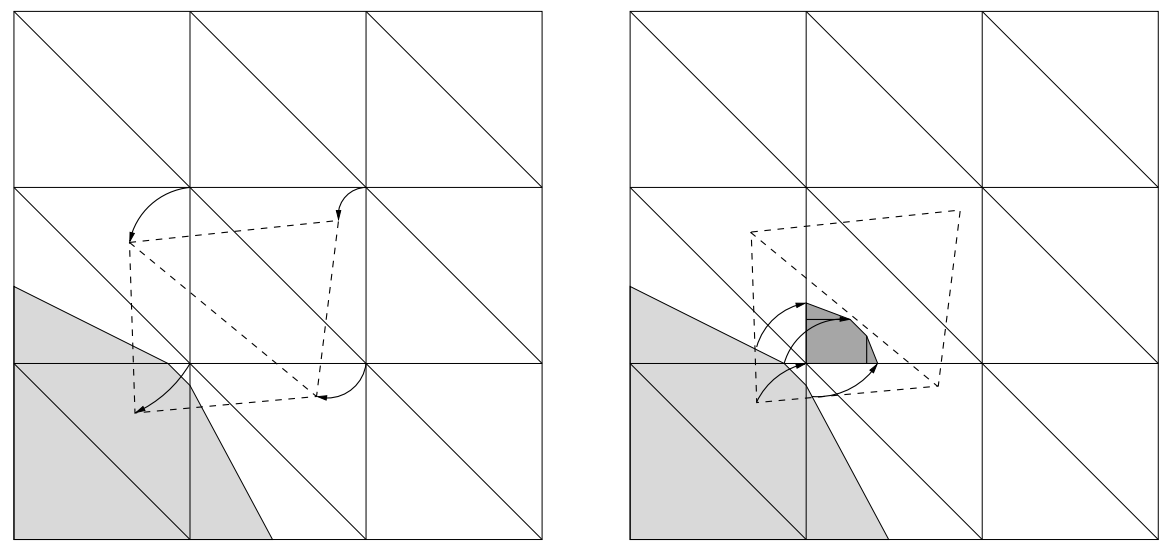

Figure 2: Left: Mesh cut by a material interface, separating two materials (light/shaded). The polygonal departure region is shown as a dotted line. Right: Material is mapped along characteristics from the departure region to the cell of interest. Mapped gridlines are shown in the target cell.

- We have found our results to be only slightly sensitive to how the target region is triangulated (see Figure 2). The results of the droplet flow test problem from section 6.3 are visually symmetric in spite of the fact that the triangulation of the target region is not symmetric. We compared our non-symmetric triangulation to a symmetric triangulation in which the target region is split into 4 triangles instead of 2 . For the reversible vortex test problem in section 6.1, with two levels of refinement, splitting the target region into 4 triangles improved the results by only 10 percent and was 50 percent slower. For the Droplet Flow test problem in section 6.3 , the symmetric triangulation of the target region improved the accuracy by only 20 percent, and was 60 percent slower.

\section{Conglomeration Algorithm}

\subsection{Basic Algorithm}

In general, the Cell-Integrated Semi-Lagrangian advection procedure maps portions of material from multiple cells to a single cell (the target cell). Each of these cells is potentially cut by an interface and 
intersected with the departure region that will be mapped to the target cell. Once all portions of the intersected cells have been mapped to the target cell, the target cell is occupied by a set of triangulated polygons. A conglomeration procedure is used to identify which triangular regions should be grouped together when reconstructing the material interface. Each triangle carries a material ID, and adjacent triangular figures with matching material ID's should be grouped into the same region, or conglomerate. The simplest algorithm for forming these material conglomerates is as follows.

1. Choose a starting triangle $T_{i}$.

2. Identify all ungrouped triangles $T_{j}$ that are adjacent to $T_{i}$ and have the same material ID.

3. If new triangles have been identified, repeat from Step 1 for each $T_{j}$. If no new triangles are identified, then continue.

4. Denote grouped triangles a completed conglomerate $C_{i}$.

5. Compute volume fraction $F_{i}$ and centroid $\boldsymbol{x}_{i}$ of conglomerate $C_{i}$.

6. Identify any ungrouped $T_{k}$. If no such triangle exists, all conglomerates have been found; exit. If there does exist such a triangle, return to Step 1.

Figures 3 - 5 shows the steps of the conglomeration procedure, given a triangulated region with material ID's. 


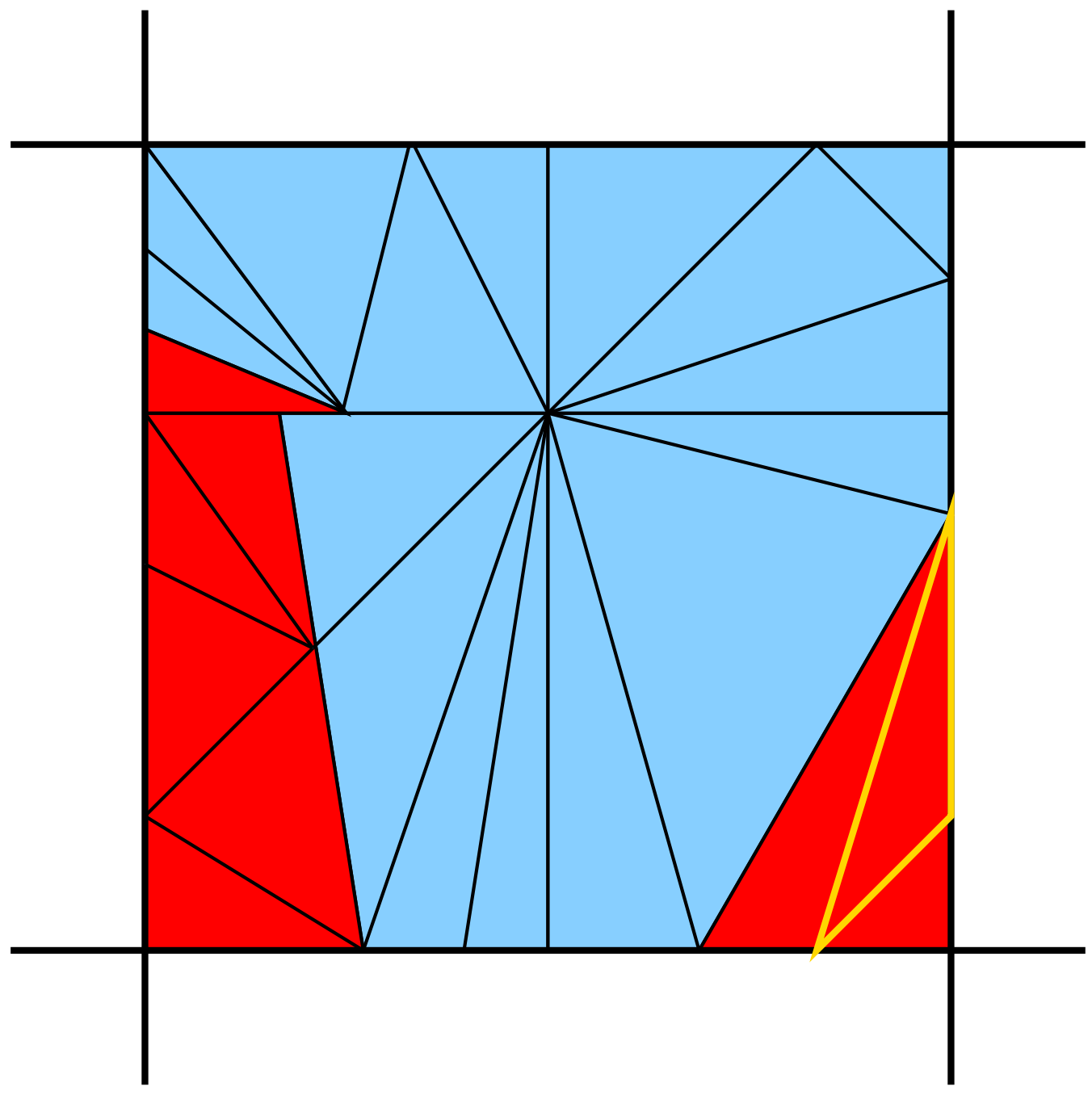

Figure 3: Rectangular cell with multiple, triangulated, polygonal regions. Different materials denoted as red and blue regions in the cell. Starting triangle $T$ in the bottom-right corner is outlined in gold. 


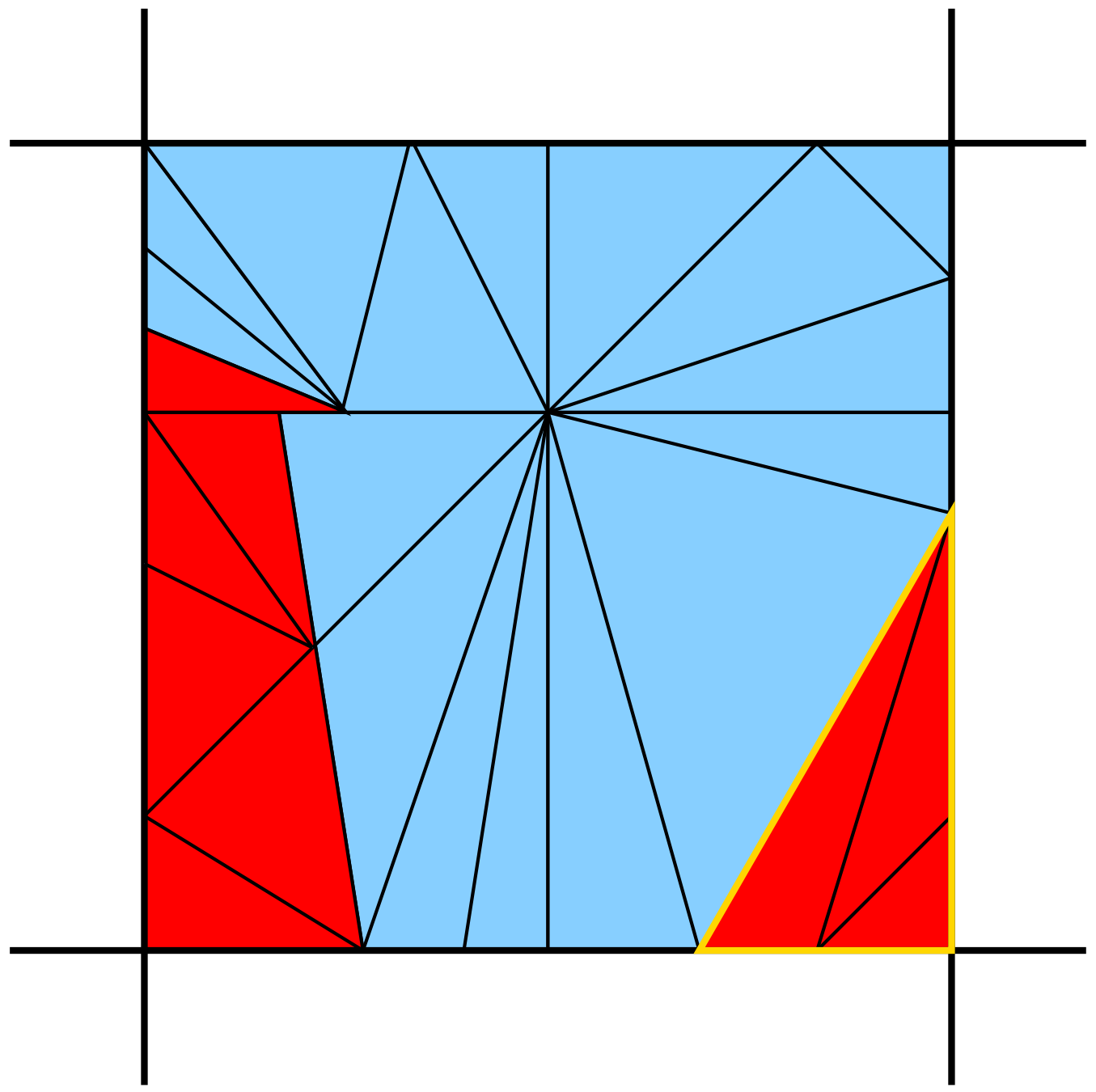

Figure 4: Adjacent neighbors to triangles in Fig. 3 grouped (in gold, bottom-right) under the conglomeration algorithm. 


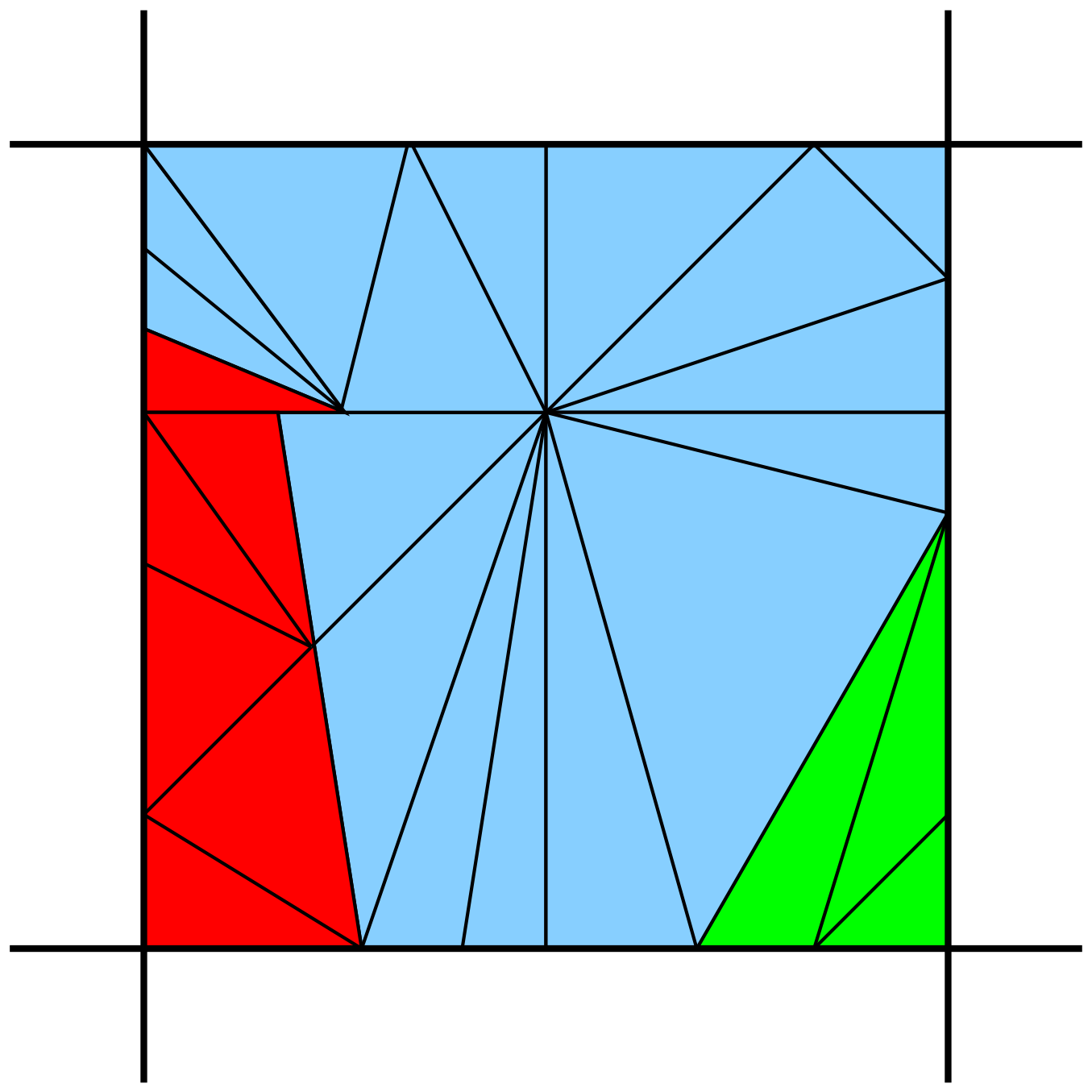

Figure 5: Cell with fictitious "twin" material introduced in green, from the conlgomerated region. 


\subsection{Adjacency}

Consider two triangles $T_{i}$ and $T_{j}$ with sides represented by line segments $S_{1, i}, S_{1, j}, S_{2, i}$, etc. For the purpose of conglomeration, triangles $T_{i}$ and $T_{j}$ are considered "adjacent" if for some pair of sides $S_{k_{1}, i}$ and $S_{k_{2}, j},(4.1)$ holds, for some open set $(a, b)$.

$$
(a, b) \subset S_{k_{1}, i} \cap S_{k_{2}, j}
$$

In essence, triangles should not be conglomerated in the case that the intersection of their sides is the empty set or a single point. $\mathrm{Nu}-$ merically, we cannot directly compare values to test for adjacency of sides, due to round-off errors that are inherent to calculation with real numbers. Instead, the sides are taken as vectors. If the cross-product between sides (4.2) falls below a tolerance, the sides are said to be parallel.

$$
\left\|\vec{S}_{k_{1}, i} \times \vec{S}_{k_{2}, j}\right\|_{\infty} \leq h^{2} \epsilon
$$

If sides are found to be parallel, then the projection of the endpoints of one side onto the linear extension of the other side is found. If the difference between the endpoint and the projection of the endpoint falls below a certain tolerance (4.3), the sides are said to be co-linear. Finally, co-linear points can be parametrized, so that one may find the intersection of the two line segments. If the intersection is some open set, the triangles are said to be adjacent.

$$
\left\|\boldsymbol{x}-\boldsymbol{x}_{\text {project }}\right\|_{1} \leq h \epsilon
$$

In all of our simulations, $h=\Delta x$ and $\epsilon=10^{-3}$.

\subsection{Conglomerate Configuration}

Once all conglomerates have been found, several situations can exist.

1. There is only one conglomerate, i.e. the cell is a pure cell with only one material.

2. There are two conglomerates with different material ID's. In this case, the algorithm reverts to the two material MOF interface reconstruction.

3. There are multiple conglomerates with different material ID's. In this case, a decision must be made about interface topology. 
In the case of a pure cell, there is nothing to be done in terms of either conglomeration or interface reconstruction because no interface is present. This can be identified by preprocessing during the conglomeration routine to improve performance. If all triangular elements have the same material ID, perform no conglomeration; write the input ID's to the output and continue. In the case of one conglomerate of each material, the cell is cut by a single interface. This is the situation for which Moment-of-Fluid Interface Reconstruction was designed. The conglomeration algorithm will perform the grouping, discover that only one conglomerate of each material exists, then write the material ID's to output. The interface reconstruction will be identical to MOF because no fictitious material ID's are introduced. This is computationally less efficient because more work will be expended with no gain in accuracy.

In the final case, multiple conglomerates of a given material exist, meaning a filament may form. Let us define a filament as a material region that separates two like-material regions such that they are not adjacent. We will denote the material that is separated by the filament as the "bulk" material. Note that the labels filament and bulk make no assumption of relative size of the regions.

If a cell is cut by a filament with interfaces of zero curvature, we wish to be able to recover the material configuration exactly. With Multimaterial Moment of Fluid, it is possible to capture the interfaces within the tolerance of volume and centroid error in the reconstruction process. By assigning a fictitious third material ID to one of the disconnected bulk regions, the procedure of nested dissection can be used to recover the two interfaces. As with a true multimaterial case, it is necessary to choose the ordering that minimizes reconstruction error. If the filament material is selected first for reconstruction, the resulting material configuration may differ significantly from the true material configuration. While the optimal ordering for reconstruction is not known a priori, experiments show that the conglomerate with the centroid farthest from the cell center is less likely to be the filamentary material. During the procedure of nested dissection, the material to be reconstructed is chosen as the material whose centroid is farthest from the uncaptured region. Shown in Fig. 6, improper material ordering can result in improper interface topology, changing from a filamentary configuration to a T-junction or triple point.

While an exhaustive search of all possible material orderings will yield the ordering that produces the lowest reconstruction error, we 
find that it is significantly faster, with little penalty in error, to use (2.7) as the criterion for selecting the material ordering. For example, checking all possible material orderings for the reversible vortex test problem in section 6.1 improved the accuracy by only one percent (number of levels equals two) but increased the run time by 60 percent. Checking all material orderings had the biggest impact for the Droplet Flow test problem in section 6.3 in which the error still decreased by only 15 percent while the run time increased by 35 percent.
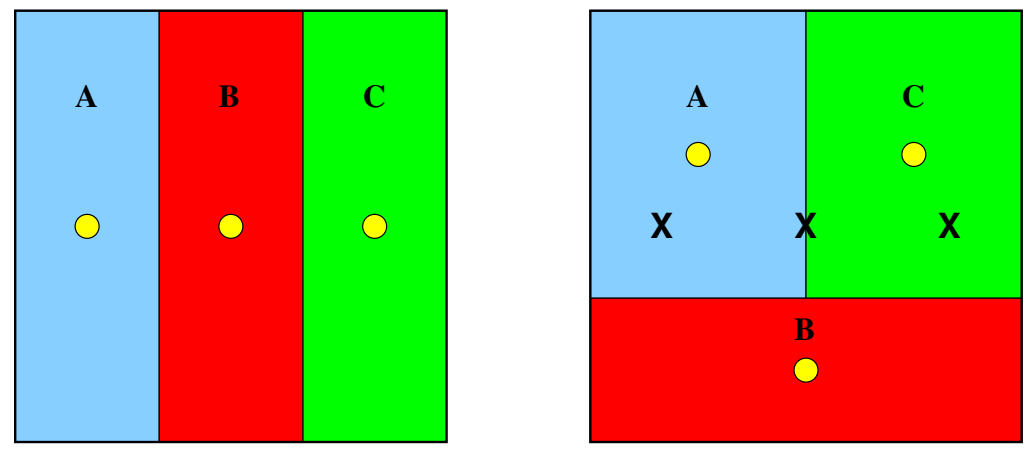

Figure 6: Left: Cell with three materials, with moments shown as a yellow circle. Right: If the improper reconstruction ordering is taken (starting with Material B), interface topology is incorrect. Reference moments are shown as a black "X;" actual moments are shown as a yellow circle.

\subsection{Disconnected Conglomerates}

A common occurrence in numerical simulations of deforming interfaces is the formation of "flotsam," small regions of material in a cell of a different material. Flotsam, typically indicative of error in the advection procedure or interface reconstruction, can separate from an interface and merge at a later time. Define flotsam as a material conglomerate such that no vertex of the conglomerate lies on the cell boundary. By this definition, flotsam cannot form a filament, as it does not divide a cell into disconnected regions. There exist several possible scenarios with regards to flotsam.

1. The flotsam is the only conglomerate of its material type in the cell.

2. There exists one other conglomerate of the same material type as the flotsam in the cell. 
3. There are multiple conglomerates of the same material type as the flotsam in the cell.
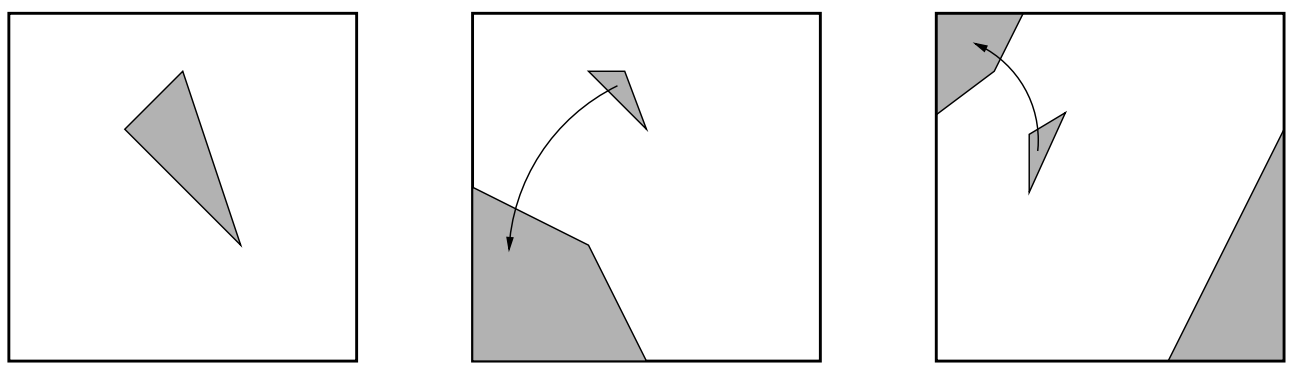

Figure 7: Left: Case 1. Flotsam in a cell. Middle: Case 2. Flotsam and one other like-material conglomerate in a cell. Right: Case 3. Flotsam with multiple material conglomerates in a cell. Arrow indicates inclusion in volume/moment calculation.

In the first case, only two material conglomerates are detected, so a filament does not form. The standard MOF interface reconstruction is used. In the second case, three material conglomerates are detected. However, logic in the conglomeration routine detects that one of the conglomerates is flotsam. The flotsam moment and volume fraction is included as is in the volume and moment calculation of the other like-material conglomerate, and the standard, two-material MOF reconstruction is used. In the final case, a filament may form. The Flotsam moment and volume fraction is included as is in the volume and moment calculation of the "nearest" like-material conglomerate, according to distance between conglomerate centroids. In the last two scenarios, the location and orientation of the interface will be affected proportionally by the volume and location of the flotsam.

Note: the flotsam in these cases cannot be accurately resolved with PLIC methods, as they are under-resolved by the grid, so we address them in a volume-conservative fashion. Re-attachment of flotsam can be interpreted as a sub-grid scale error in the Lagrangian transport phase, where flotsam material is perturbed by a position of order $\Delta x$, as in Cases 2 and 3 in Figure 7. For all 3 cases, if the reconstruction error is sufficiently large, then AMR will be triggered. However, the volume fractions of flotsam material relative to cell volume is typically $\ll 1$, which results in negligible overall interface reconstruction error. 


\subsection{General Conglomerate Algorithm}

Consider now the general case, with multiple conglomerates of two materials. We seek to identify "optimal" groupings in the sense that the conglomerates formed can best be represented by one or two interfaces. The full conglomeration algorithm is presented.

1. Form all Material 1 and Material 2 conglomerates: 1, 2, 1', 2', $1 ", 2 ", 1^{(3)}$, etc.

2. Choose at most the two largest conglomerates of each material that are adjacent to a cell edge. These are the candidates to be the bulk material. If one material has no conglomerates adjacent to the cell edge (i.e. all conglomerates are interior to the cell), use the standard MOF reconstruction and skip to the final step.

3. Attach all remaining conglomerates to the nearest like-material bulk candidate.

4. Introduce fictitious material ID's to account for any material with more than one conglomerate. Up to 4 materials may exist in a cell: $1,2,1$ ', 2'. Primes denote a fictitious material ID.

5. Compute reference volumes and moments.

6. If two conglomerates of each material exist, then two separate interface reconstructions must be performed; continue to the next step. Otherwise, perform an interface reconstruction as in Multimaterial MOF and skip to the final step.

7. Perform an interface reconstruction assuming that Material 1 is the bulk material and Material 2 is the filament. Perform another reconstruction assuming that Material 2 is the bulk material and Material 1 is the filament.

8. Accept the interface reconstruction that minimizes centroid error.

Assigning remaining conglomerates to the "nearest" bulk candidate is performed based on distances between conglomerate centroids. The fictitious fluid labels allow for differentiation between disconnected conglomerates of the same material. Multimaterial MOF will interpret them as different fluids and perform the dissection and reconstruction procedure accordingly. This is different from the procedure of standard MOF. During computation of volumes and moments of the materials in the cell in the standard Moment-of-Fluid procedure, only the material ID is taken into account when grouping polygonal 
elements. With no respect to the adjacency of like-material elements in a cell, all polygonal elements of the same material will be grouped into a single conglomerate, to use the language of this paper. In a two material simulation, this will result in two conglomerates in all mixed cells, regardless of the topology of the material configuration.

If a filament forms and generates a new, fictitious material ID, this does not prevent material from reforming at a later time step to a single material, eliminating the fictitious material ID. Prior to the detection of pure cells, a preprocessing step is carried out; all secondary, fictitious material ID's are replaced by the primary material ID before the conglomeration procedure begins. Grouping does not differentiate between a material and its fictitious counterpart that was assigned a fictitious label at a previous time step. Physically, they represent the same material, and they should be grouped together if adjacent.

\section{Time Stepping on an Adaptive Grid}

It is possible to capture a filament by introducing a fictitious material and performing a MOF interface reconstruction for three materials. However, the case when the tip of a filament enters a cell is best captured using Adaptive Mesh Refinement (AMR). Adaptive Mesh Refinement uses the error in the reconstruction as a metric to refine the grid. To accurately capture the material configuration near the tip of a filament, a modification to the AMR procedure is used.

\subsection{Mesh Initialization \& Dynamic Regridding}

Conglomeration is performed in each cell at time $t=0$, and the interface reconstruction is performed. The MOF error in each cell is computed over all materials $m$. The MOF error accounts for material volume $\left(V^{m}\right)$ and the difference between the input, material reference centroid $\left(\boldsymbol{x}_{r e f}^{m}\right)$ and the actual reconstructed material centroid $\left(\boldsymbol{x}_{a c t}^{m}\right)$.

$$
E_{M O F}=\sum_{m} V^{m}\left\|\boldsymbol{x}_{r e f}^{m}-\boldsymbol{x}_{a c t}^{m}\right\|
$$

For each cell, a $3 \times 3$ stencil, or "super cell," is then considered. See Figures 8 and 9 . The conglomeration procedure and interface reconstruction are carried out again over the super cell. The error over this 
"super cell" $\left(E_{M O F}^{S}\right)$ is recomputed, as in (5.2).

$$
E_{M O F}^{S}=\sum_{m} V^{m, S}\left\|\boldsymbol{x}_{r e f}^{m, S}-\boldsymbol{x}_{a c t}^{m, S}\right\|
$$

If the error in the "super cell" exceeds the error tolerance and the maximum level of refinement has not been reached, the cell is tagged for refinement. Additionally, cells in a narrow buffer zone are tagged for refinement. Tagged cells, along with cells in the buffer zone, are collected to form the refined grid. This procedure is then repeated on the refined grid, recomputing the reconstructed interface on the fine mesh and recalculating the MOF error.

This method for capturing the tips of filaments allows for the dynamic formation of these sharp tips. There are two scenarios: (A) there is a tip at $t=0$, and AMR is triggered; (B) there is no tip in the beginning, but a tip forms due to deformation by the flow. In both cases (A) and (B), AMR is triggered automatically. In the latter case, the AMR grids are dynamically adjusted, using the error indicators (5.1-5.2). This dynamic regridding procedure is performed at every time step.
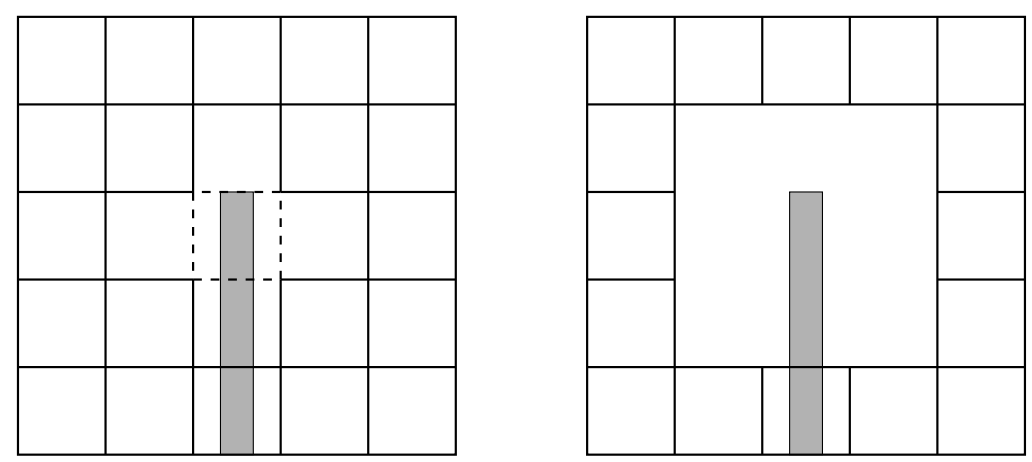

Figure 8: Left: A $5 \times 5$ grid with the tip of a filament. Filament material is shaded; bulk material is in white. Right: The $3 \times 3$ "super cell," shown embedded in the grid, for the central cell (dotted) in the $5 \times 5$ mesh. The enlarged cell will yield only two material conglomerates, resulting in a large reconstruction error $E_{M O F}^{S}$. Note that even in the case that the tip of the filament aligns with the mesh, AMR will be triggered. It is assumed that during the subsequent advection step, the tip will enter a new cell and the additional resolution will be necessary. 

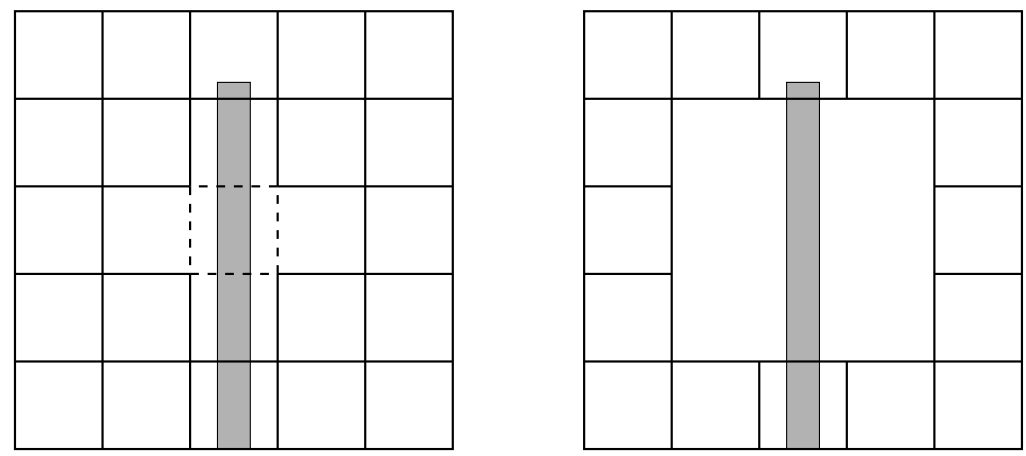

Figure 9: Left: A $5 \times 5$ grid with the tip of filament. Right: The $3 \times 3$ "super cell," shown embedded in the grid around the center cell (dotted). The enlarged cell will yield three material conglomerates. This results in a low reconstruction error $E_{M O F}^{S}$ because the filament is well-resolved in both the fine mesh cell and enlarged $3 \times 3$ cell, so AMR is not needed at the center (dotted) cell. However, the cell above the dotted cell (near the filament tip) will trigger AMR.

Near the tip of a filament, error in a given cell may be small, but error in the "super cell" will be large (Fig. 8). This is due to the fact that in the $3 \times 3$ enlarged cell, the tip of the filament does not divide the cell into three distinct regions. During the conglomeration procedure, only two distinct material regions will be detected, so no filament will be introduced. This results in a large MOF error. If error is small in the cell and the $3 \times 3$ enlarged cell (Fig. 9), then the cell is far enough away from the tip that mesh refinement is not needed. Mesh refinement (Fig. 10) is essential to accurately capture the interface as the tip of the filament enters a new cell.

Note: Work was undertaken to eliminate the need for Adaptive Mesh Refinement at the tips of filaments by modeling tips as a "wedge," i.e. two intersecting linear interfaces with degrees of freedom specified as angular offset from and linear displacement along a central axis. In principle, this could exactly capture sharp corners, while also modeling filament tips. However, this introduced an optimization problem that was computationally expensive and produced results that were not reliably accurate. Additionally, developing a uniform detection criterion for introducing this machinery was found to be difficult. While a filament can be detected through the geometric procedures described in Section 4, the tip of a filament does not subdivide a cell into distinct, 


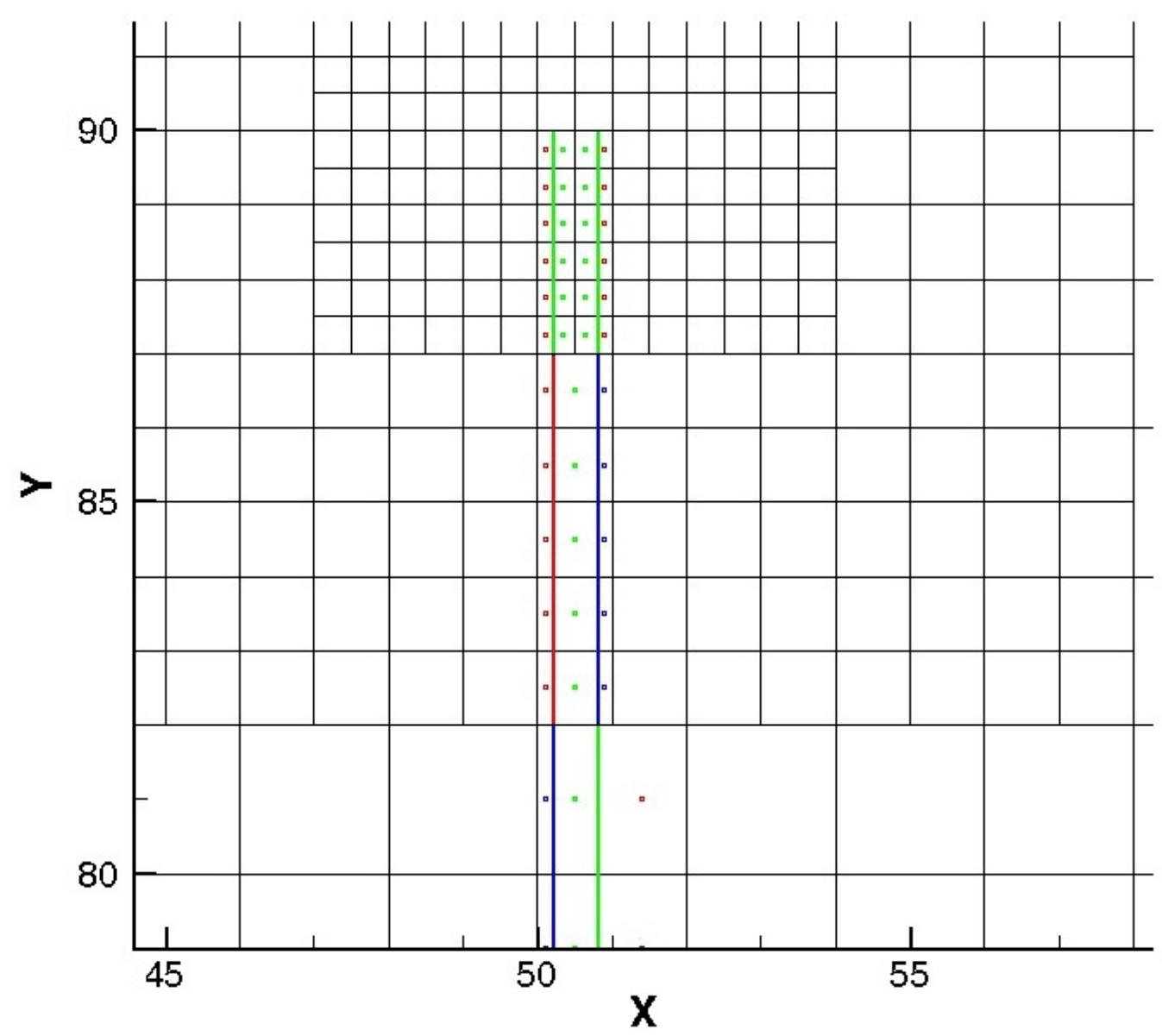

Figure 10: Filament tip with two levels of Adaptive Mesh Refinement. Cells are refined when error in the $3 \times 3$ super cell exceeds the AMR error tolerance. Additionally, cells in a narrow band about a refined cell are tagged for refinement as well. Centroids and interfaces are shown for Material 1 (red), Material 2 (green), and fictitious Material 1' (blue).

separated regions of material (Fig. 11). Rather, detection of the tip of a filament would require a finely tuned error parameter. It was determined that use of AMR was preferable to attempting to resolve these issues. 


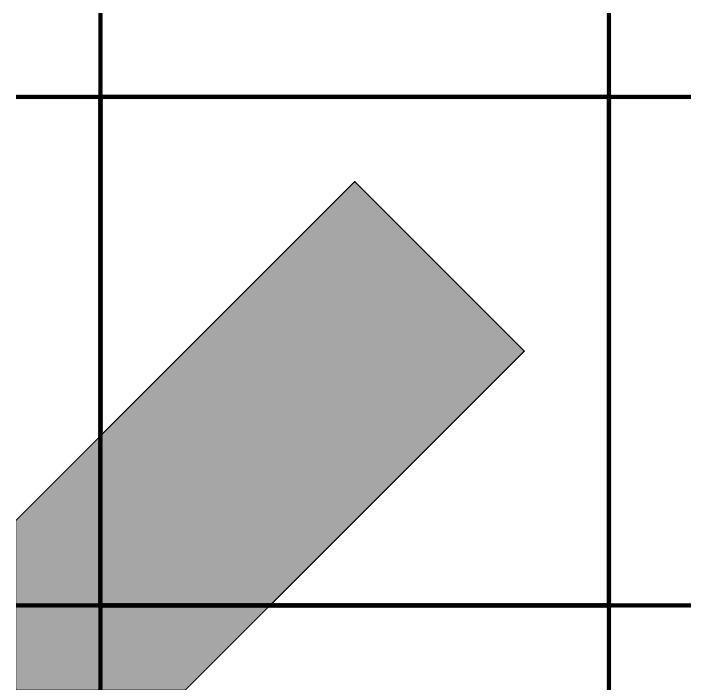

Figure 11: A filament tip entering a cell does not subdivide the cell into distinct regions, making it difficult to detect and capture this case. Adaptive Mesh Refinement can be applied successfully to a number of material configurations.

\subsection{Coarse-To-Fine Data Interpolation}

Where fine level grid cells are adjacent to coarse level grid cells, fine level "ghost cells" are formed using coarse level data. Using the reconstructed interface $\Gamma_{i}$ from the coarse cell, the interface is reconstructed in the fine level ghost cells by cutting each fine level cell with $\Gamma_{i}$ (Fig. 12) 

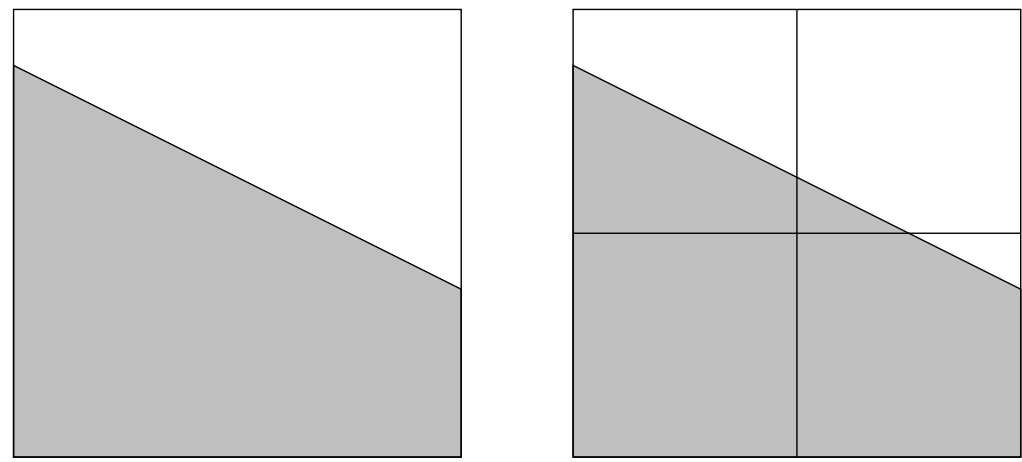

Figure 12: Left: Coarse cell cut by an reconstructed interface $\Gamma_{i}$. Right: Coarse cell data interpolated to fine cells. Each fine cell is cut by $\Gamma_{i}$.

\subsection{Data Synchronization}

Data is synchronized between fine levels and coarse levels using the following procedure, starting at the finest level.

1. Compute the slope reconstruction.

2. If the level is less than the maximum level of refinement, use the "average down" procedure (Fig. 13 - 14).

3. Compute the MOF error in conjunction with the conglomeration algorithm to account for filaments. 

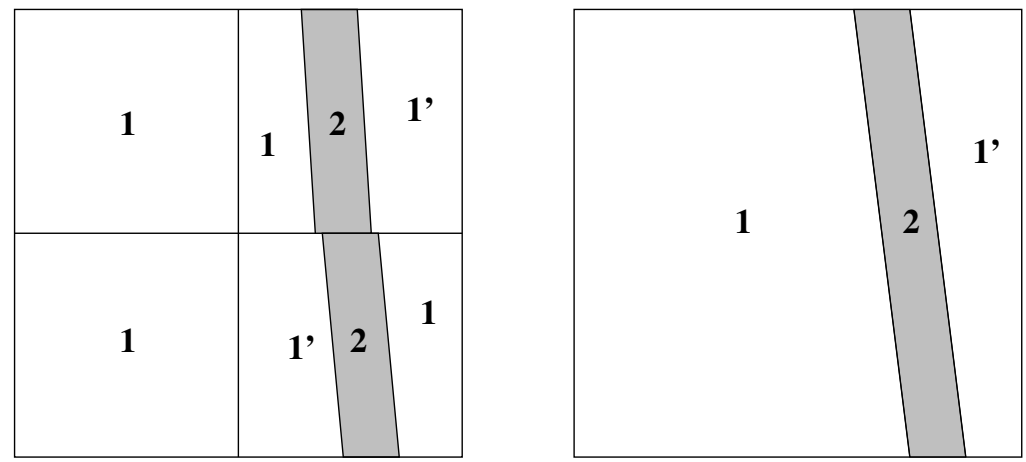

Figure 13: Illustration for the synchronization of fine to coarse filamentary data. Left: A $2 \times 2$ stencil of fine level cells cut by a filament. Right: The corresponding coarse cell with resolved filament. The conglomeration procedure performs a grouping of the materials 1 and $1^{\prime}$ in the $2 \times 2$ fine grid stencil in order to create a new grouping in the underlying coarse cell with Material 1 on the left side of the filament and Material $1^{\prime}$ on the right side of the filament.
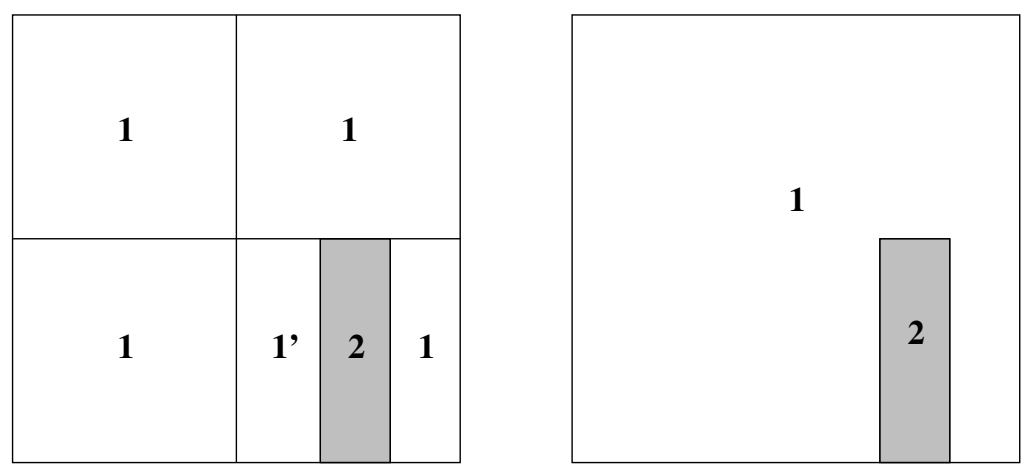

Figure 14: Left: A $2 \times 2$ stencil of fine level cells cut by a filament. Right:

The corresponding coarse cell with unresolved filament. The conglomeration procedure at the coarse level will detect only two conglomerates. No filament will be introduced, leading to a large reconstruction error in the coarse cell.

\subsection{Time Stepping}

After the material configuration on the mesh has been initialized, the procedure of advection and interface reconstruction can be carried out. 
1. Multimaterial slope reconstruction is performed.

2. The Cell-Integrated Semi-Lagrangian procedure advects material. When the departure region for a fine-level cell intersects a coarse-level cell, coarse-level data is interpolated to fine-level data, as in Section 5.2. Filaments in the advective preimage of a cell are detected and resolved using the conglomeration and reconstruction procedure.

3. Data is synchronized between fine levels and coarse levels, as in Section 5.3.

4. The refined grid is dynamically regridded.

(a) Starting at the coarsest level $L$, tag cells with MOF error exceeding the threshold.

(b) Group tagged cells, along with cells in the error buffer, to generate the next finest grid level, $L+1$.

(c) Starting at level 0, generate the new grid level. Use the error indicator and either (i) interpolate from a previously existing level or copy data from a coarser level.

\section{$6 \quad$ Numerical Results}

For all of the numerical results that we report, the adaptive mesh is regenerated every time step, the adaptive mesh refinement buffer size is two cells, and the blocking factor for grids on a given level is two. In other words, for each cell that is tagged to be adapted, we also tag the neighboring cells whose index is within 2 cells of the tagged cell. Rectangular grids at the next adaptive level are created such that all tagged cells are covered by a finer rectangular grid and the number of cells on a side of any grid is divisible by 2 .

The criterion for tagging a cell is that the MOF error (5.1) or the super cell MOF error (5.2) exceeds $0.0003 \Delta x$ where $\Delta x$ is the mesh size on a given level.

\subsection{Reversible Vortex}

The initial material configuration is defined by a circle of radius $\mathrm{R}$ $=0.15$ with center $(0.50,0.75)$ located within the unit square domain $[0,1] \times[0,1]$. The circular region is deformed by a non-linear, unsteady velocity field given by stream function (6.1). 


$$
\psi(x, y, t)=\frac{1}{\pi} \sin ^{2}(\pi x) \sin ^{2}(\pi y) \cos \left(\frac{\pi t}{T}\right)
$$

Parameter $T$ defines the time for the full period of the flow field, with larger $T$ leading to greater deformation. For the long period reversible vortex, we take $T=8$. Time $t=T$ would see the analytical solution returning to the initial material configuration. Time $t=\frac{T}{2}$ defines the time of maximum material deformation.

We report the symmetric difference error (6.2) for our simulations, with $\Omega_{E}$ and $\Omega_{C}$ representing the exact and computed material configurations, respectively. Symmetric difference error and performance statistics at full-reversal are shown in Tables 1 through 3. At coarser grid levels, when the number of cells marked for refinement is comparable between the Standard MOF and MOF with filament capturing, the additional overhead of the conglomeration procedure leads to a larger runtime. Faster runtime for MOF with filaments is observed when two levels of AMR are used, because there are fewer Level 2 grid cells (compare Figure 15 to Figure 17). Additionally, thin structures are preserved without breakup when using filament capturing (compare Figure 16 to Figure 18).

The advantage of using filament capturing is seen in the gains in accuracy at a given mesh refinement level. The symmetric difference error (6.2) when using filament capturing with a given number of levels of mesh refinement is slightly lower than the error calculated when using the "filament detection logic disabled" MOF algorithm with an additional level of mesh refinement.

$$
E_{S D}=\left|\left(\Omega_{E} \cup \Omega_{C}\right) /\left(\Omega_{E} \cap \Omega_{C}\right)\right|
$$




\begin{tabular}{|c|c|c|c|}
\hline & AMR Level & Error & Runtime (sec) \\
\hline \multirow{2}{*}{ Filament MOF } & 0 & $3.12 \times 10^{-3}$ & 32.6 \\
& 1 & $6.91 \times 10^{-4}$ & 200.0 \\
& 2 & $2.77 \times 10^{-4}$ & 635.3 \\
\hline \multirow{2}{*}{ MOF (filament disabled) } & 0 & $1.99 \times 10^{-2}$ & 28.7 \\
& 1 & $4.82 \times 10^{-3}$ & 183.3 \\
& 2 & $3.34 \times 10^{-4}$ & 804.5 \\
\hline \multirow{2}{*}{ Ahn \& Shashkov [2] } & 0 & $2.33 \times 10^{-2}$ & N/A \\
& 1 & $3.15 \times 10^{-3}$ & N/A \\
& 2 & $5.04 \times 10^{-4}$ & N/A \\
\hline
\end{tabular}

Table 1: Results for the long-period deforming vortex at full reversal. Base grid mesh resolution is $32^{2}$. The MOF algorithm without filament detection takes longer than the filament MOF algorithm when number of AMR levels is 2 because the moment error is large in filamentary regions and requires a larger patch of the computational domain to be fully adapted. We also compare the symmetric difference error for this problem with the standard volume fraction error $((14)$ in [2]) reported by [2] for the same problem using the MOF method with quad-tree AMR (instead of block structured

AMR) and a fixed time step of $\Delta t=1 / 32$ (instead of a variable $\Delta t$ determined from the CFL condition).

\begin{tabular}{|c|c|c|}
\hline & AMR Level & Cells Advanced \\
\hline \multirow{2}{*}{ Filament MOF } & 0 & $1.26 \times 10^{6}$ \\
& 1 & $2.01 \times 10^{6}$ \\
& 2 & $9.26 \times 10^{5}$ \\
\hline \multirow{2}{*}{ MOF (filament disabled) } & 0 & $1.31 \times 10^{6}$ \\
& 1 & $3.08 \times 10^{6}$ \\
& 2 & $4.25 \times 10^{6}$ \\
\hline
\end{tabular}

Table 2: Cells advanced on each level when using 2 levels of AMR in the long-period deforming vortex test. Comparison is made between using filament capturing and the MOF algorithm with filament detection logic disabled. The non-filament MOF algorithm advances approximately 50\% more Level 1 cells and over 350\% more Level 2 cells than using the filament machinery. This supports the observation that MOF with filaments takes less time to run at 2 levels of AMR than the MOF without filaments. Base grid mesh resolution is $32^{2}$. 


\begin{tabular}{|c|c|c|}
\hline & Max. AMR Level & Cells Advanced \\
\hline \multirow{2}{*}{ Filament MOF } & 0 & $3.35 \times 10^{5}$ \\
& 1 & $1.62 \times 10^{6}$ \\
& 2 & $4.19 \times 10^{6}$ \\
\hline \multirow{2}{*}{ MOF (filament disabled) } & 0 & $3.35 \times 10^{6}$ \\
& 1 & $2.06 \times 10^{6}$ \\
& 2 & $8.64 \times 10^{6}$ \\
\hline
\end{tabular}

Table 3: Total number of cells advanced on all levels for various levels of mesh refinement in the long-period deforming vortex test. As mesh refinement is increased, significantly fewer cells are advanced when using the filament method, as compared to the MOF method with filament detection disabled. 


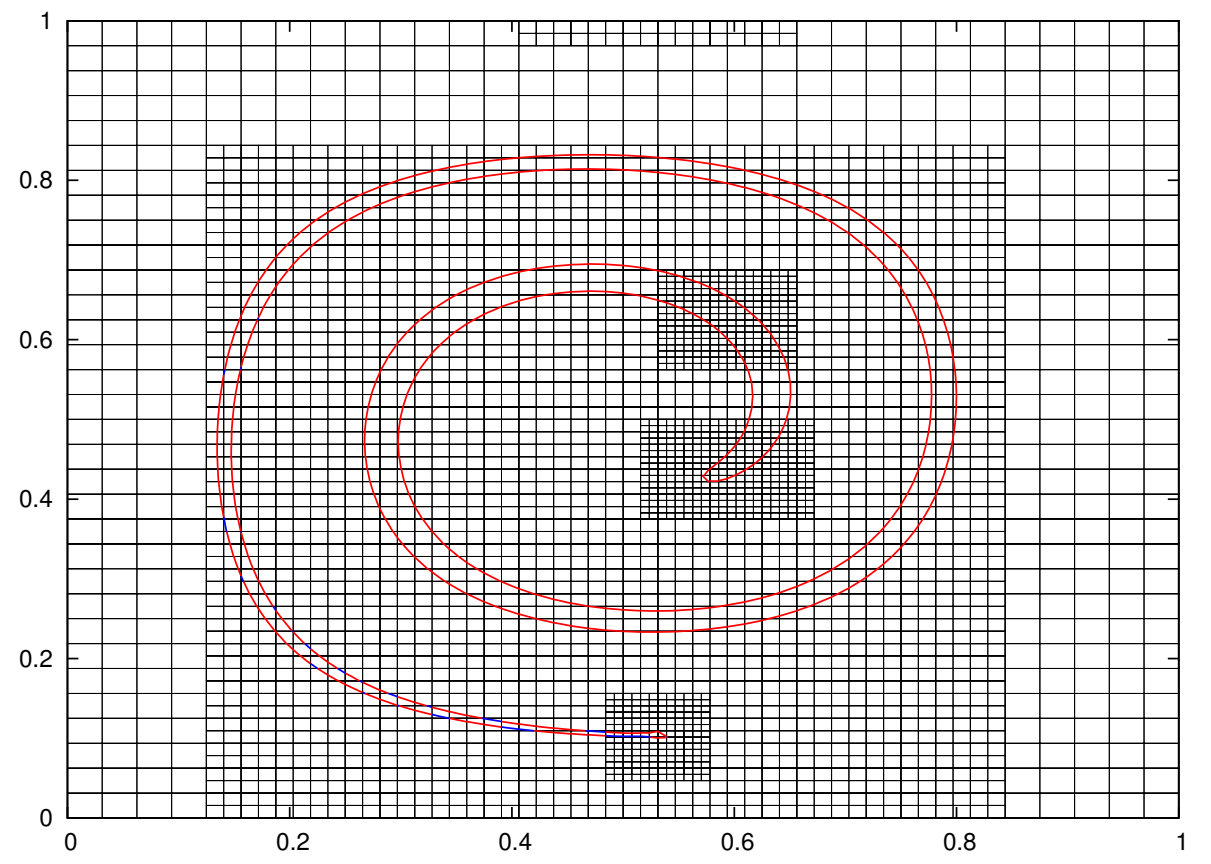

Figure 15: Reversible vortex shown at maximum deformation for $N=32$ grid resolution and 2 levels of AMR with filament machinery. The blue segments represent the interface between material 1 and material 2 . The red segments represent the interface between the "fictitious" third material and material 2. This fictitious third material is introduced when non-adjacent groups of the same material are detected. The Moment of Fluid method is then used to reconstruct the material configuration as a problem with three materials and two interfaces, rather than two materials with a single interface. 


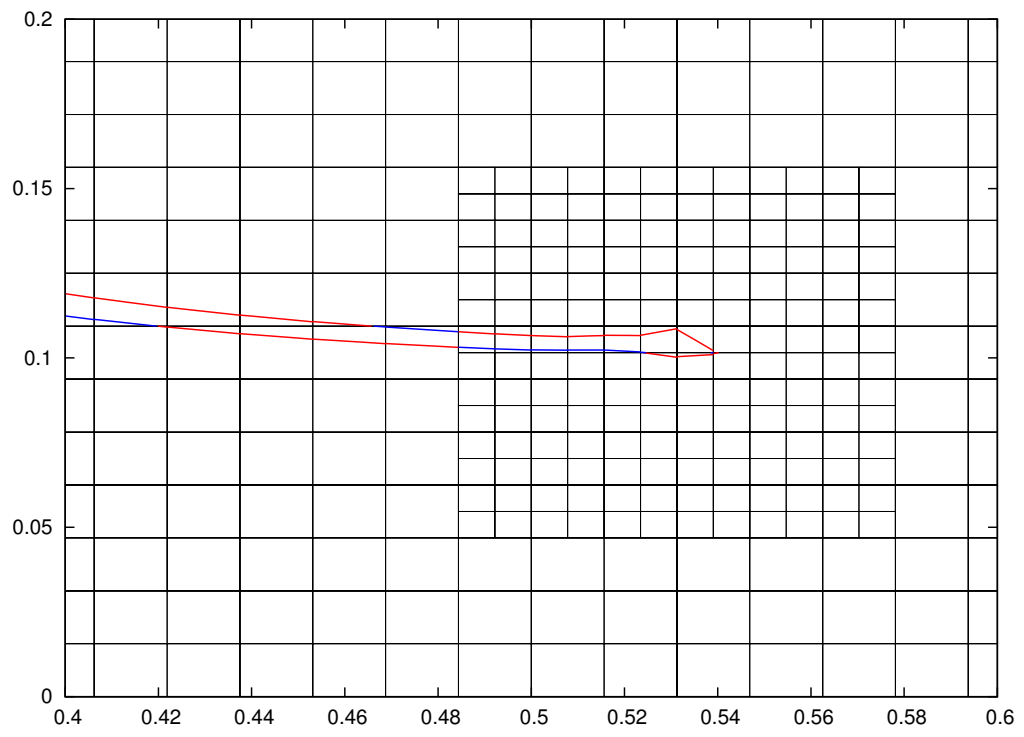

Figure 16: Reversible vortex shown at maximum deformation for $N=32$ grid resolution and 2 levels of AMR with filament machinery, zoomed at the thin tail of the interface. Note that the thin structure is accurately captured. 


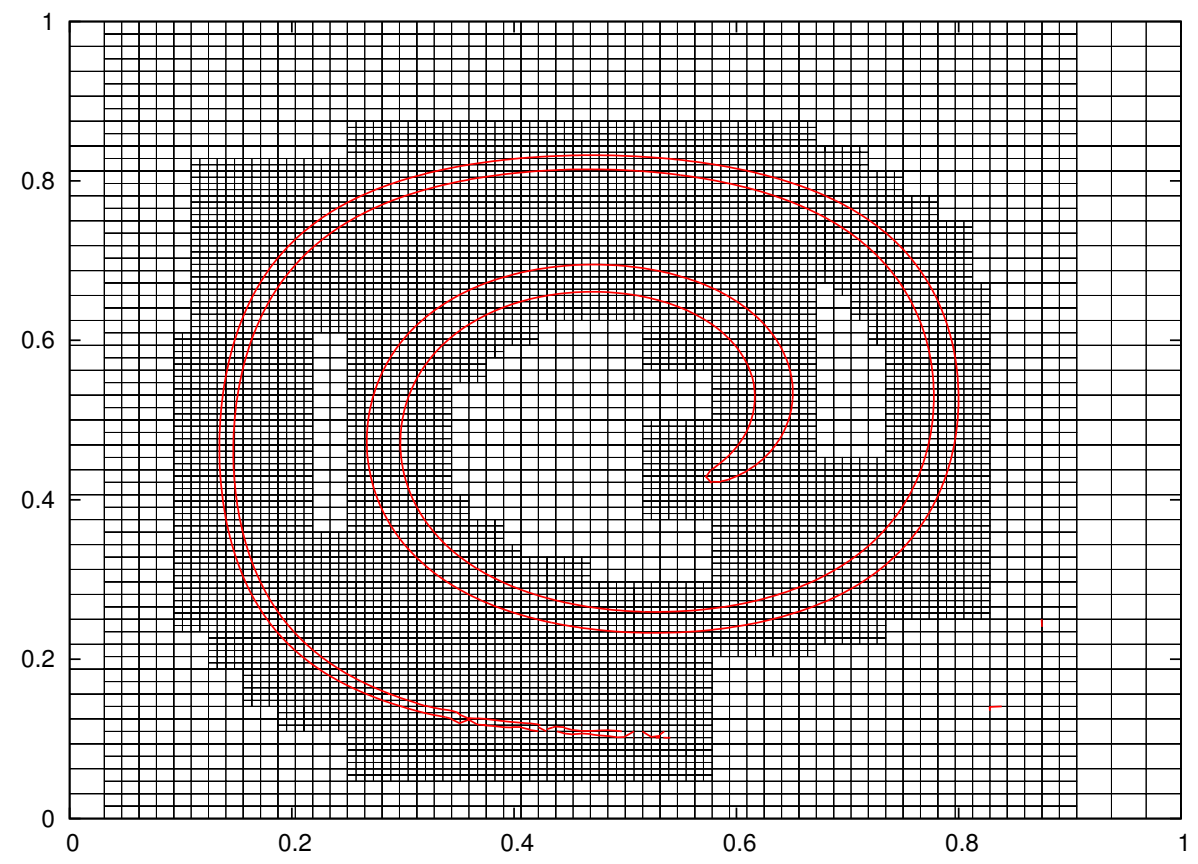

Figure 17: Reversible vortex shown at maximum deformation for $N=32$ grid resolution and 2 levels of AMR using the Moment of Fluid method with the filament logic disabled. The red segments represent the interface between material 1 and material 2. Note that much more of the grid is refined than when using MOF with filament capturing, leading to longer runtime for the same effective resolution. 


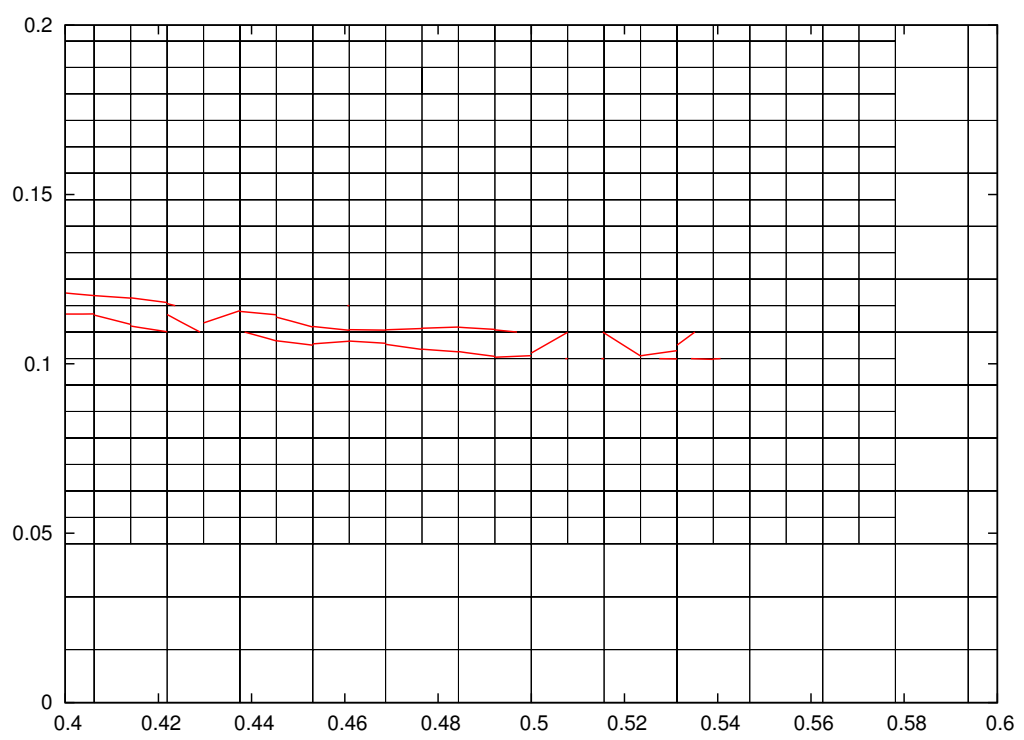

Figure 18: Reversible vortex shown at maximum deformation for $N=32$ grid resolution and 2 levels of AMR using the Moment-of-Fluid method with the filament logic disabled. The figure is zoomed in at the thin tail of the interface in order to illustrate the numerical break-up of the thin structure. 


\subsection{Rotating Filament}

A finite, rectangular filament of length 30 and width 0.6 is initialized with center at the point $(50.5,75)$ in the domain $\Omega=[0,100]^{2}$. The filament is initially oriented vertically. A velocity field is applied to rotate the filament counter-clockwise about the center of the domain. Initial mesh resolution is $50 \times 50$. The top and bottom ends of the filament are initially aligned with the mesh. Two levels of AMR are used to resolve the tips of the filament, using the procedure described in Section 5.1. Note that while the center of the filament is underresolved by the mesh, AMR is not triggered because the reconstruction error is below the AMR error threshold.

Figure 19 shows the filament after completing $1 / 8$ of its revolution. Notice that no breakup is exhibited, even though the filament is underresolved by the mesh. AMR is triggered by reconstruction errors only in a narrow region around the tips of the filament. Figures $20-21$ show the tip of the filament before and after completing one revolution. Error in the reconstruction, manifested as mass diffusion near the tip of the filament, is due to the inability to exactly resolve a corner within a cell. 


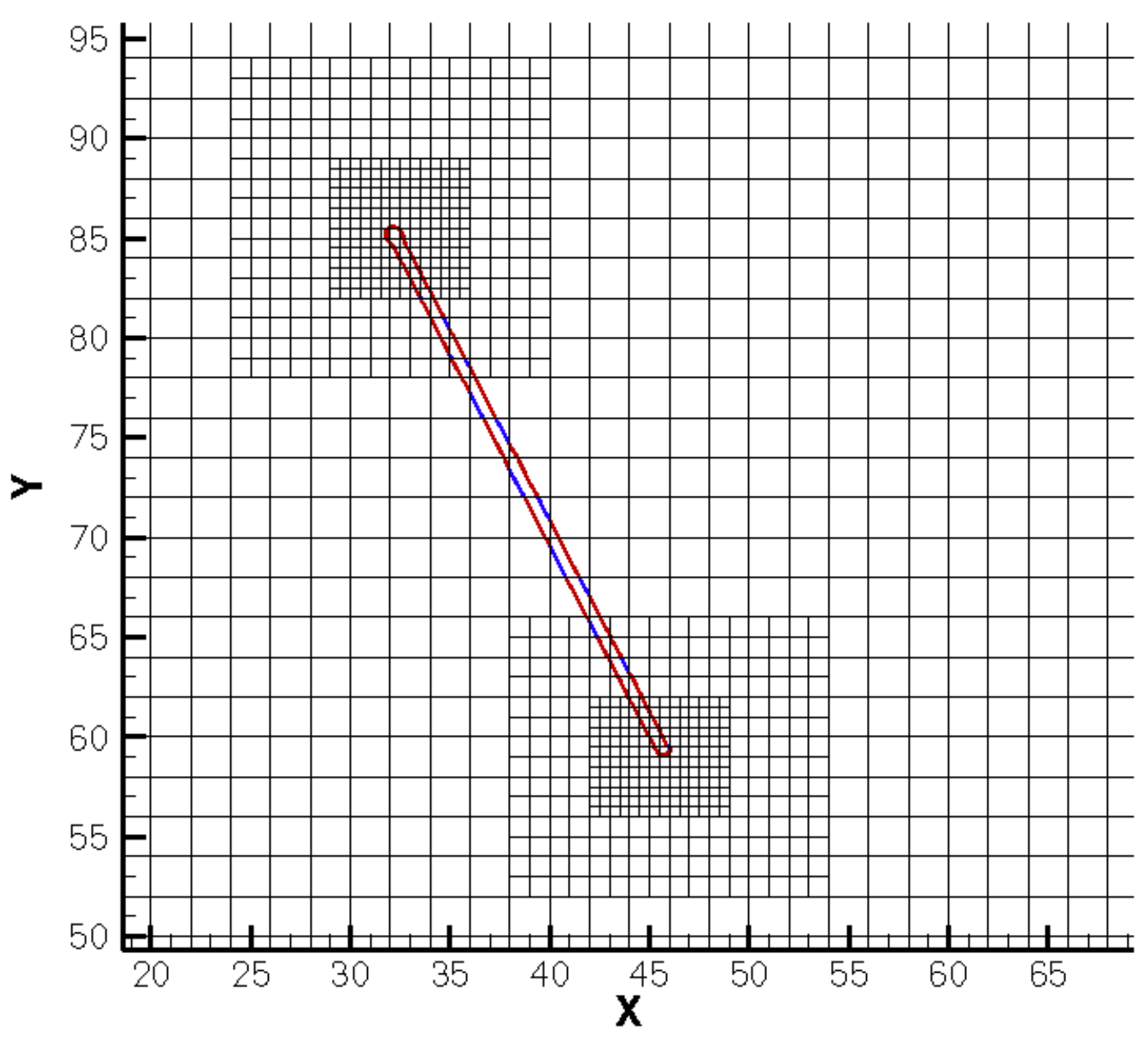

Figure 19: Rotating filament after $1 / 8$ of a revolution. A blue interface indicates the introduction of a fictitious material to capture a filament in the cell. Two levels of AMR are used to resolve the tips of the filament. 


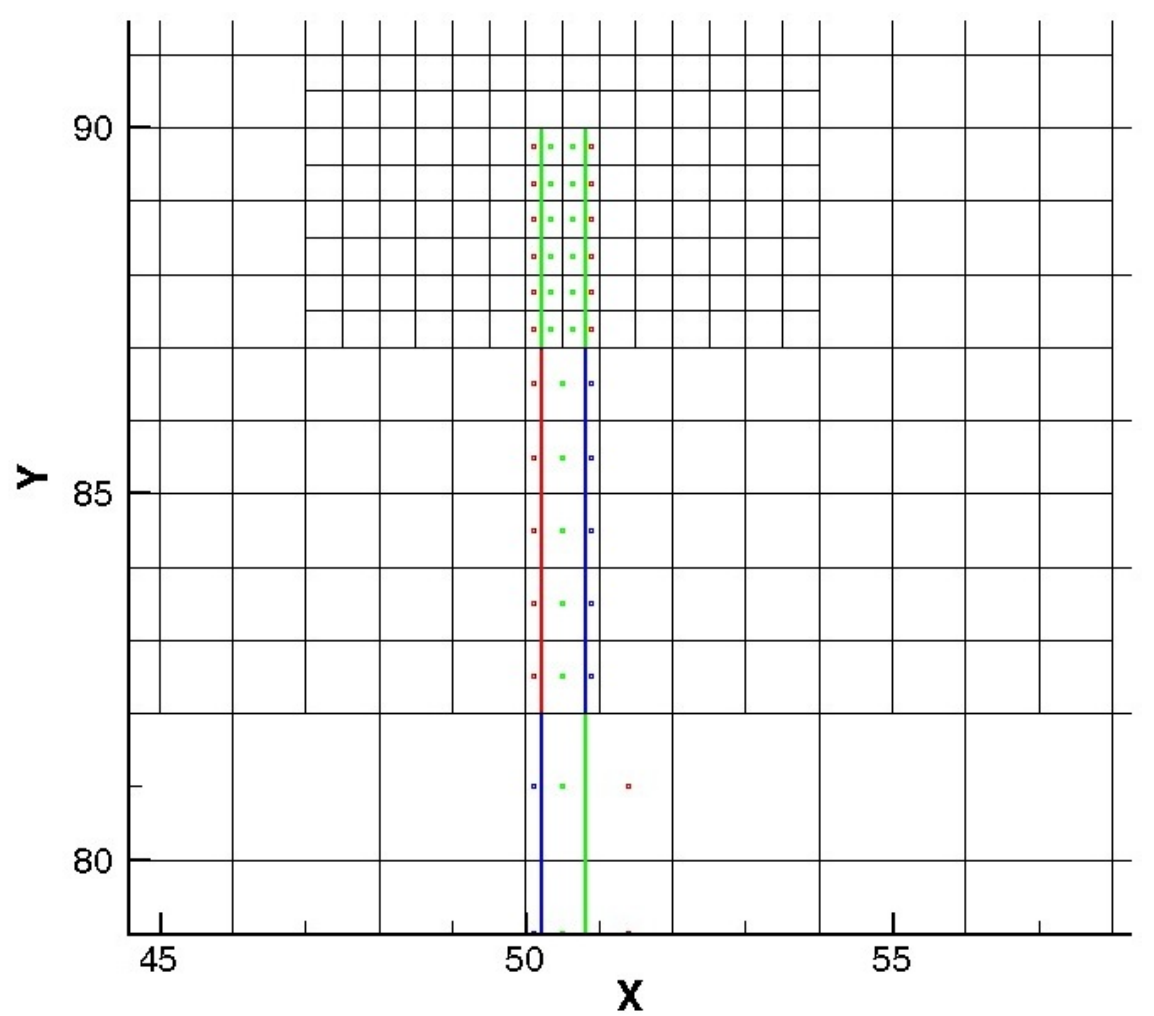

Figure 20: The tip of the filament before revolution. Interfaces and centroids are shown for each material. 


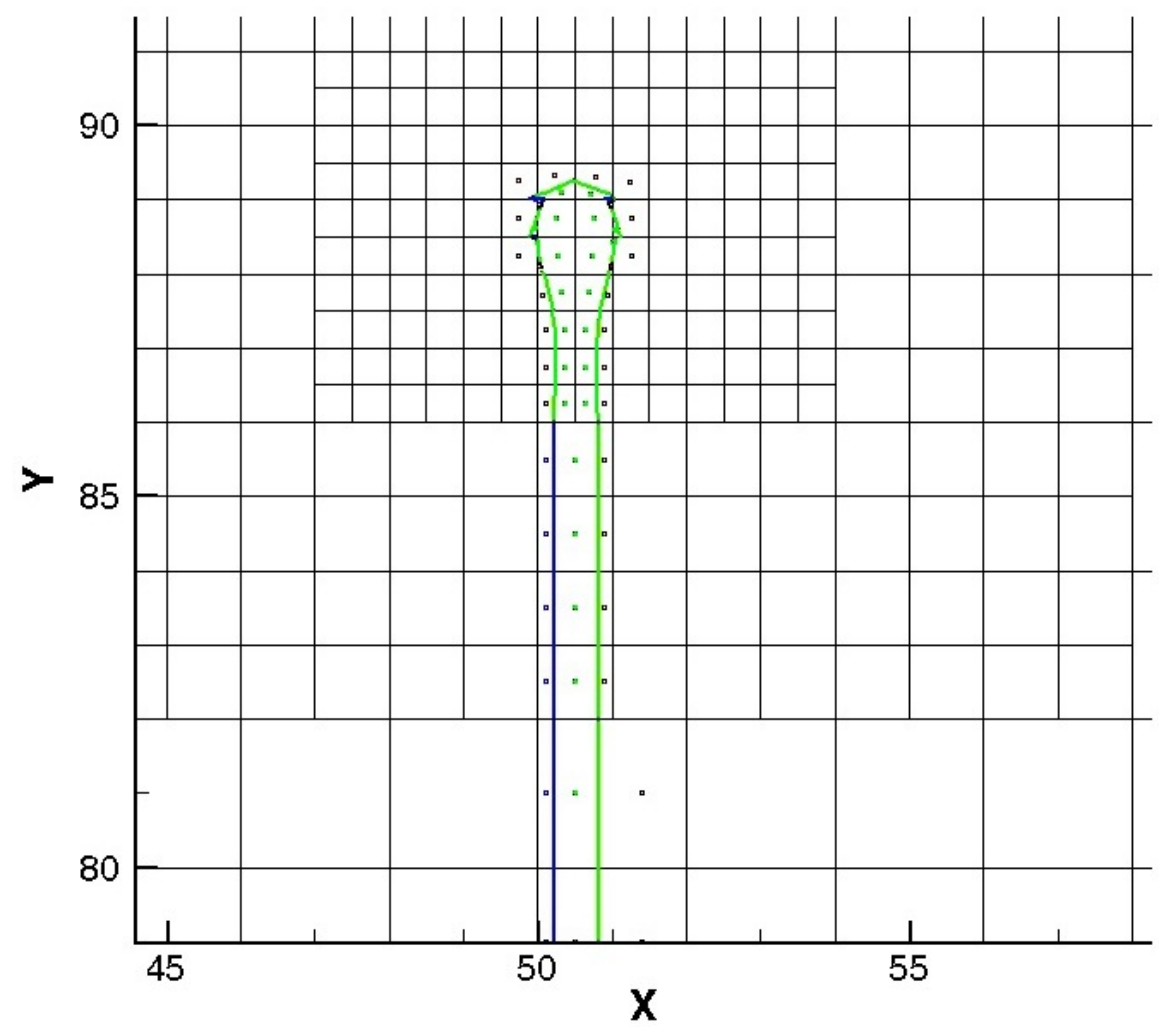

Figure 21: The tip of the filament after revolution. Diffusion of mass has occurred near the tip, but the body of the filament is accurately captured. 


\subsection{Droplet Flow}

A nonlinear, divergence-free velocity field acts upon a circular region, causing edges to shear and form a filament. At time $t=0$, the circular region is located in the center of the $[0,1]^{2}$ domain, with initial radius $r_{0}=0.125$. This test case, introduced in [2], presents a different challenge from the test case in Section 6.1. In this test case, filaments form as the circular region is deformed into a droplet shape with two sharp edges. The filaments at these sharp tips must be accurately captured to prevent spurious breakup (see Figures 22 - 26). Mesh refinement is also applied at the bottom of the droplet, as the curvature of the droplet can lead to a non-negligible reconstruction error when using a piecewise-linear interface.

The flow induces deformation until time $T_{\text {maxdef }}=0.8$, at which point the flow is reversed, and the simulation continues until the end of one full period $T=2 T_{\text {maxdef }}$. The process of flow reversal occurs over the transition time $T_{\text {maxdef }}-t_{\epsilon} / 2 \leq t \leq T_{\text {maxdef }}+t_{\epsilon} / 2 ; t_{\epsilon}=0.1$. The following reversible velocity field is used, with error (see Tables 4 through 6) calculated at full reversal when the exact solution is the initial material configuration, a circle.

$$
\begin{gathered}
\boldsymbol{u}(x, y, t)=\left[\begin{array}{l}
\frac{1}{8}(8 x-4) \\
\left.\frac{1}{8}\left\{-(8 y-4)-4-\left(1-(8 x-4)^{2}-(8 x-4)^{4}\right)\right\}\right] f(t)
\end{array}\right. \\
f(t)= \begin{cases}1, & 0 \leq t<T_{\text {maxdef }}-t_{\epsilon} / 2 \\
\cos \left(\frac{\pi\left(t-T_{\text {maxdef }}+t_{\epsilon} / 2\right)}{t_{\epsilon}}\right), & T_{\text {maxdef }}-t_{\epsilon} / 2 \leq t \leq T_{\text {maxdef }}+t_{\epsilon} / 2 \\
-1 & T_{\text {maxdef }}+t_{\epsilon} / 2<t \leq T\end{cases}
\end{gathered}
$$




\begin{tabular}{|c|c|c|c|}
\hline & AMR Level & Error & Runtime (sec) \\
\hline \multirow{2}{*}{ Filament MOF } & 0 & $2.48 \times 10^{-3}$ & 191.3 \\
& 1 & $6.37 \times 10^{-4}$ & 529.3 \\
& 2 & $2.96 \times 10^{-4}$ & 940.4 \\
\hline \multirow{3}{*}{ MOF (filament disabled) } & 0 & $7.44 \times 10^{-3}$ & 170.4 \\
& 1 & $6.78 \times 10^{-3}$ & 473.5 \\
& 2 & $7.13 \times 10^{-4}$ & 972.4 \\
\hline
\end{tabular}

Table 4: Results for the droplet flow test case at full reversal. Base grid mesh resolution is $32^{2}$.

\begin{tabular}{|c|c|c|}
\hline & AMR Level & Cells Advanced \\
\hline \multirow{2}{*}{ Filament MOF } & 0 & $3.00 \times 10^{6}$ \\
& 1 & $3.23 \times 10^{6}$ \\
& 2 & $2.47 \times 10^{6}$ \\
\hline \multirow{2}{*}{ MOF (filament disabled) } & 0 & $3.00 \times 10^{6}$ \\
& 1 & $4.25 \times 10^{6}$ \\
& 2 & $4.26 \times 10^{6}$ \\
\hline
\end{tabular}

Table 5: Cells advanced on each level when using 2 levels of AMR in the droplet test. Comparison is made between the MOF method using filament capturing and the MOF method with filament capturing disabled. Without the filament capturing logic, approximately 30\% more Level 1 cells and approximately $75 \%$ more Level 2 cells are advanced than using the filament machinery. This supports the observation that the MOF method with filament capturing takes less time to run at 2 levels of AMR than the MOF method without filament capturing. The base grid mesh resolution is $32^{2}$. 


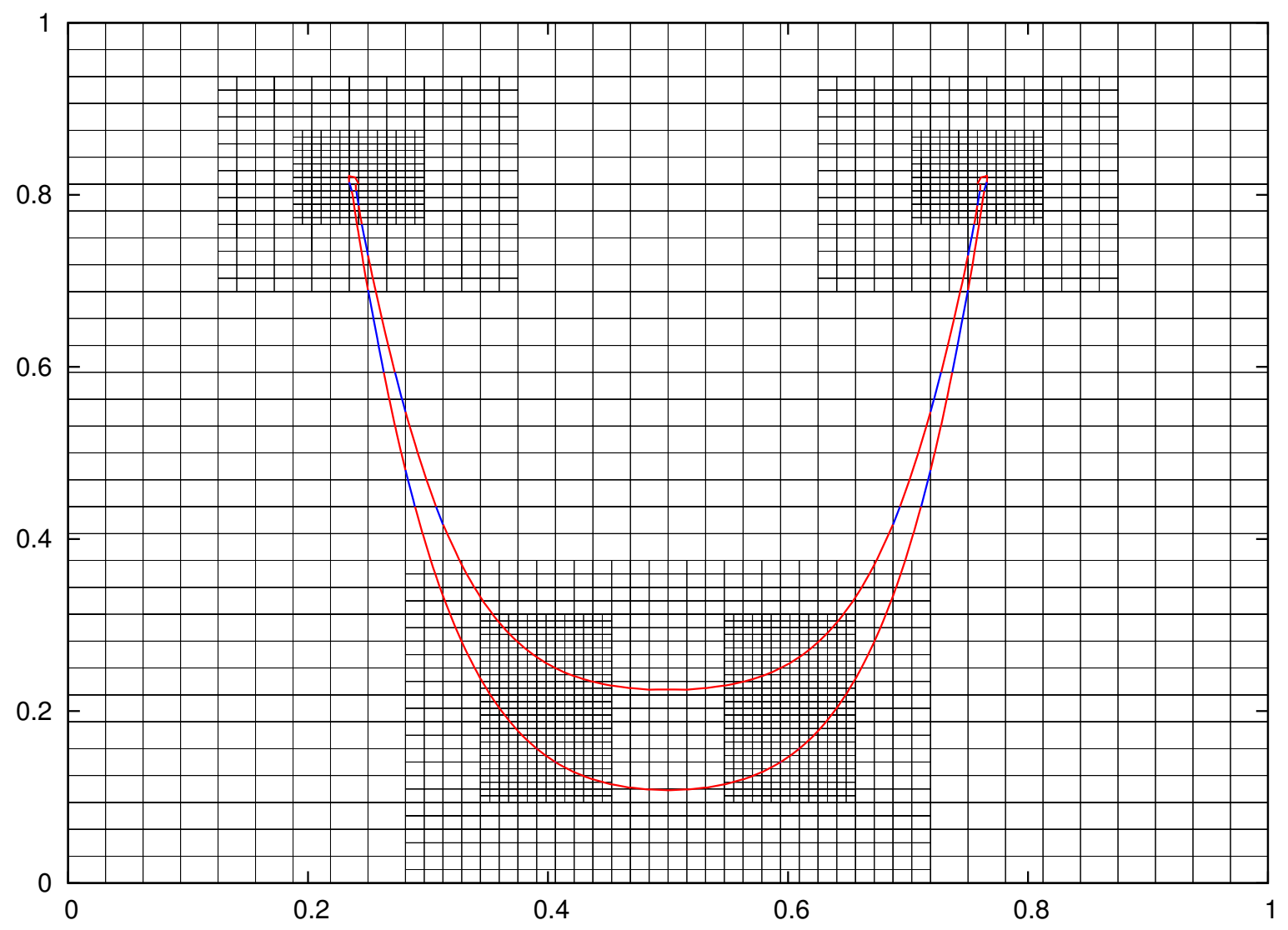

Figure 22: Droplet flow at maximum deformation, using filament capturing, with 2 levels of AMR. 

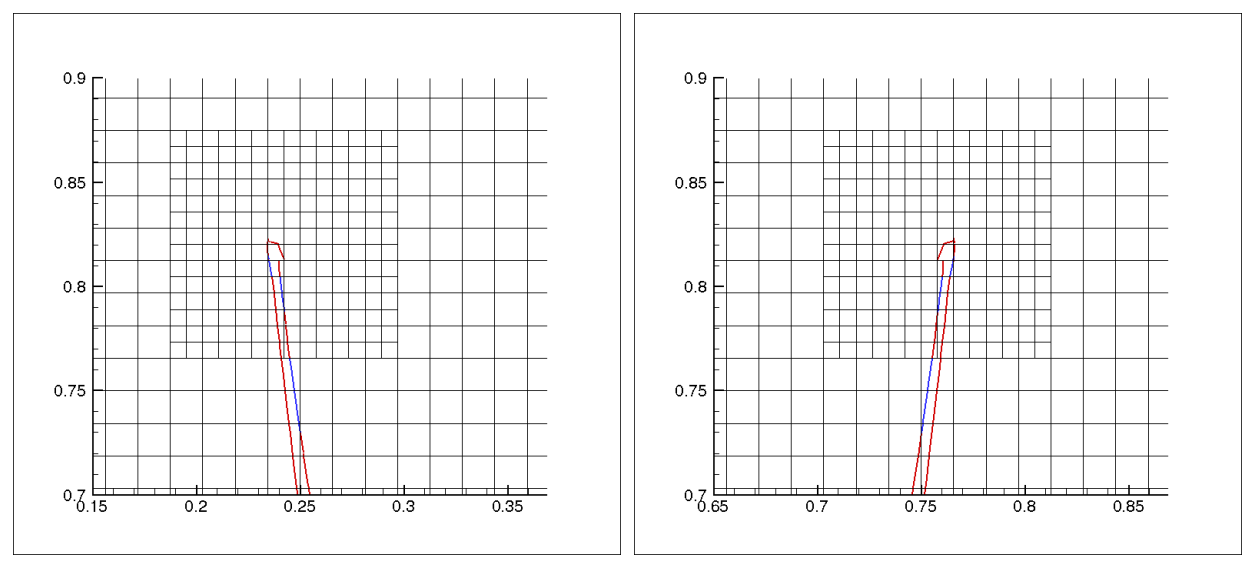

Figure 23: Droplet flow at maximum deformation, using filament capturing, with 2 levels of AMR. The view at the sharp tips is enlarged. Note that the thin structure is captured without break-up, although numerical diffusion causes the tips to become less sharp.
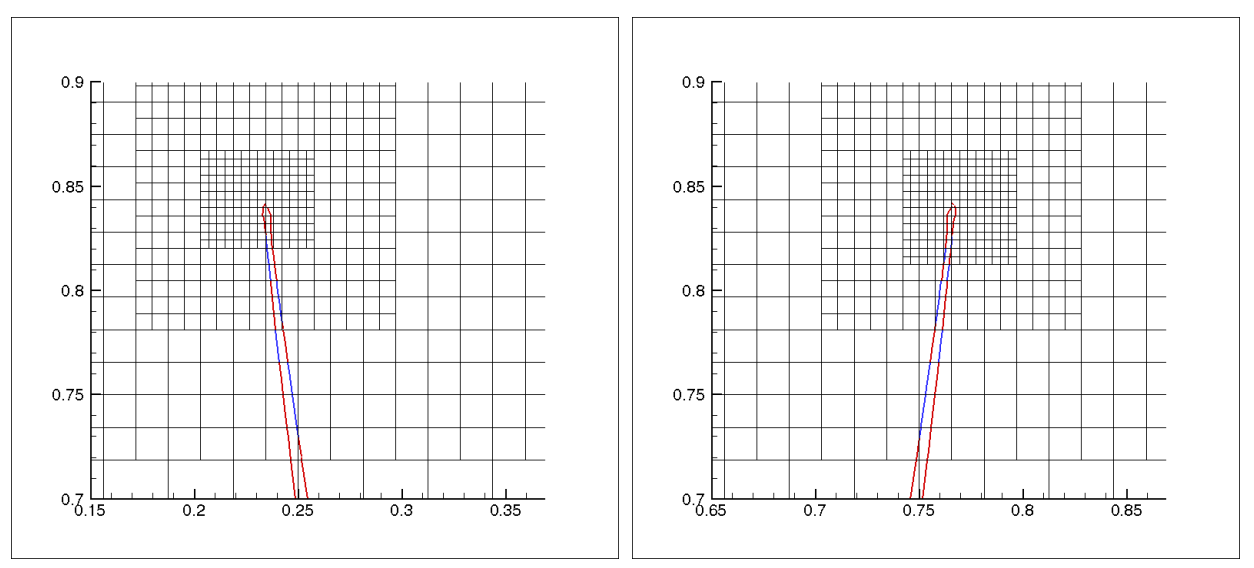

Figure 24: Droplet flow at maximum deformation, using filament capturing, with 3 levels of AMR. The view at the sharp tips is enlarged. Note that the thin structure is captured without break-up, and the tip structure is better resolved than with 2 levels of AMR. 


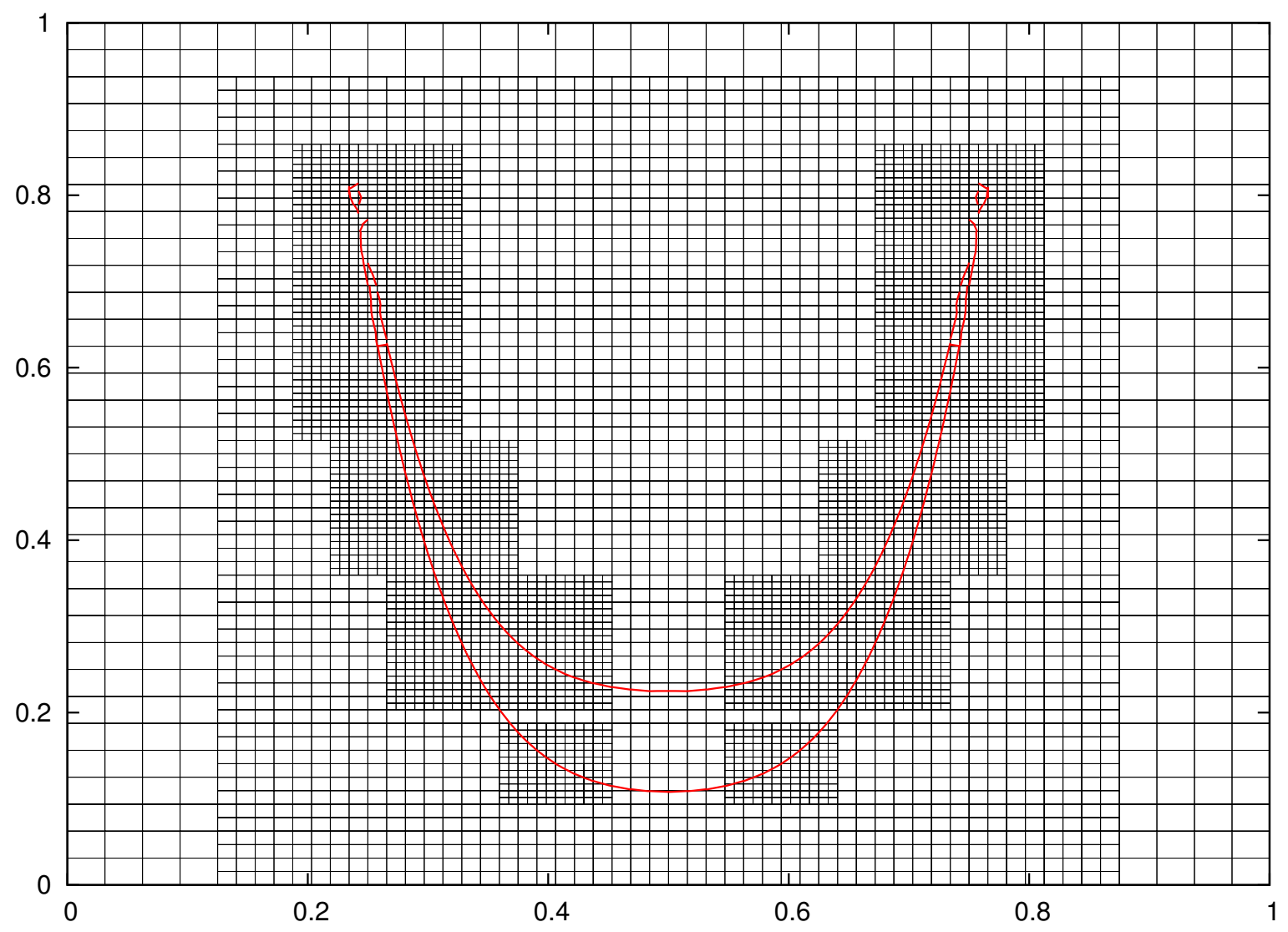

Figure 25: Droplet flow at maximum deformation, using the MOF method with the filament capturing logic disabled; 2 levels of AMR. 

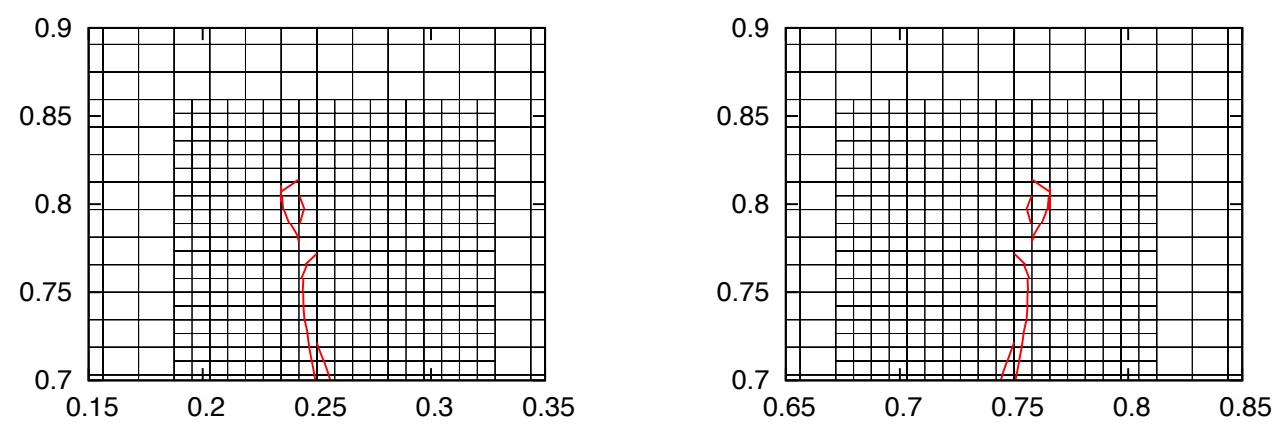

Figure 26: Droplet flow at maximum deformation, using the MOF method with the filament capturing logic disabled; 2 levels of AMR. The view at the sharp tips is enlarged. Note the irregularity in the thin structure near the tips.

\begin{tabular}{|c|c|c|}
\hline & Max. AMR Level & Cells Advanced \\
\hline \multirow{2}{*}{ Filament MOF } & 0 & $3.00 \times 10^{6}$ \\
& 1 & $5.77 \times 10^{6}$ \\
& 2 & $8.70 \times 10^{6}$ \\
\hline \multirow{2}{*}{ MOF (filament disabled) } & 0 & $3.00 \times 10^{6}$ \\
& 1 & $6.48 \times 10^{6}$ \\
& 2 & $1.15 \times 10^{7}$ \\
\hline
\end{tabular}

Table 6: Total number of cells advanced on all levels for various levels of mesh refinement in the droplet test. As mesh refinement is increased, fewer cells are advanced when using the filament method, as compared to the MOF method with filament capturing disabled. 


\subsection{S-shape Flow}

A nonlinear, divergence-free velocity field acts on a circular region, initially located at the center of a $[0,1]^{2}$ domain with radius $r_{0}=0.25$. The velocity field, introduced in [2], causes the circular region to rollup into an S-shape. This test case presents the challenge that filaments of each material are formed.

The following reversible velocity field is used, with parameters $T_{\text {maxdef }}=4.0, t_{\text {epsilon }}=2.0$, and $T=8.0$. Function $f(t)$ defining velocity field reversal is

$$
\boldsymbol{u}(x, y, t)=\left[\begin{array}{c}
\frac{1}{4}\left\{(4 x-2)+(4 y-2)^{3}\right\} \\
\left.\frac{-1}{4}\left\{(4 y-2)+(4 x-2)^{3}\right)\right\}
\end{array}\right] f(t)
$$

Again, use of filament capturing produces error results comparable to using the "filament capturing disabled" Moment-of- Fluid method with an additional level of mesh refinement, while the increase in cost associated with filament capturing at a given grid resolution is much lower than adding an additional level of refinement (see Tables 7 through 9) . Note that at one level of mesh refinement (Figures 27 and 31), filaments of each material are formed. This configuration is resolved when using filament capturing (Figures 27 and 29), while spurious break-up is observed when filament capturing is not applied (Figures 30 and 31). When 2 levels of mesh refinement is used (Figure 28 ), the extra mesh resolution makes it so that twin materials are not required as frequently.

\begin{tabular}{|c|c|c|c|}
\hline & AMR Level & Error & Runtime (sec) \\
\hline \multirow{2}{*}{ Filament MOF } & 0 & $2.11 \times 10^{-2}$ & 157.2 \\
& 1 & $1.34 \times 10^{-3}$ & 773.1 \\
& 2 & $4.74 \times 10^{-4}$ & 1871.5 \\
\hline \multirow{3}{*}{ MOF (filament disabled) } & 0 & $6.38 \times 10^{-2}$ & 138.7 \\
& 1 & $2.07 \times 10^{-2}$ & 760.2 \\
& 2 & $9.63 \times 10^{-4}$ & 2044.3 \\
\hline
\end{tabular}

Table 7: Results for the S-shape at full reversal. The base grid mesh resolution is $32^{2}$. 


\begin{tabular}{|c|c|c|}
\hline & AMR Level & Cells Advanced \\
\hline \multirow{3}{*}{ Filament MOF } & 0 & $2.27 \times 10^{6}$ \\
& 1 & $4.75 \times 10^{6}$ \\
& 2 & $4.72 \times 10^{6}$ \\
\hline \multirow{2}{*}{ MOF (filament disabled) } & 0 & $2.49 \times 10^{6}$ \\
& 1 & $6.58 \times 10^{6}$ \\
& 2 & $1.09 \times 10^{7}$ \\
\hline
\end{tabular}

Table 8: Cells advanced on each level when using 2 levels of AMR in the S-shape test. Comparison is made between using the MOF filament capturing method and the MOF method with filament capturing disabled. Approximately $40 \%$ more Level 1 cells and approximately $130 \%$ more Level 2 cells are advanced when the filament capturing logic is disabled. This supports the observation that MOF with filaments takes less time to run at 2 levels of AMR than the MOF method with filament logic disabled. The base grid mesh resolution is $32^{2}$.

\begin{tabular}{|c|c|c|}
\hline & Max. AMR Level & Cells Advanced \\
\hline \multirow{2}{*}{ Filament MOF } & 0 & $1.15 \times 10^{6}$ \\
& 1 & $5.36 \times 10^{6}$ \\
& 2 & $1.17 \times 10^{7}$ \\
\hline \multirow{2}{*}{ MOF (filament disabled) } & 0 & $1.15 \times 10^{6}$ \\
& 2 & $7.37 \times 10^{6}$ \\
& 2 & $2.00 \times 10^{7}$ \\
\hline
\end{tabular}

Table 9: Total number of cells advanced on all levels for various levels of mesh refinement in the S-shape test. As mesh refinement is increased, fewer cells are advanced when using the MOF filament method, as compared to the MOF method with filaments disabled. 


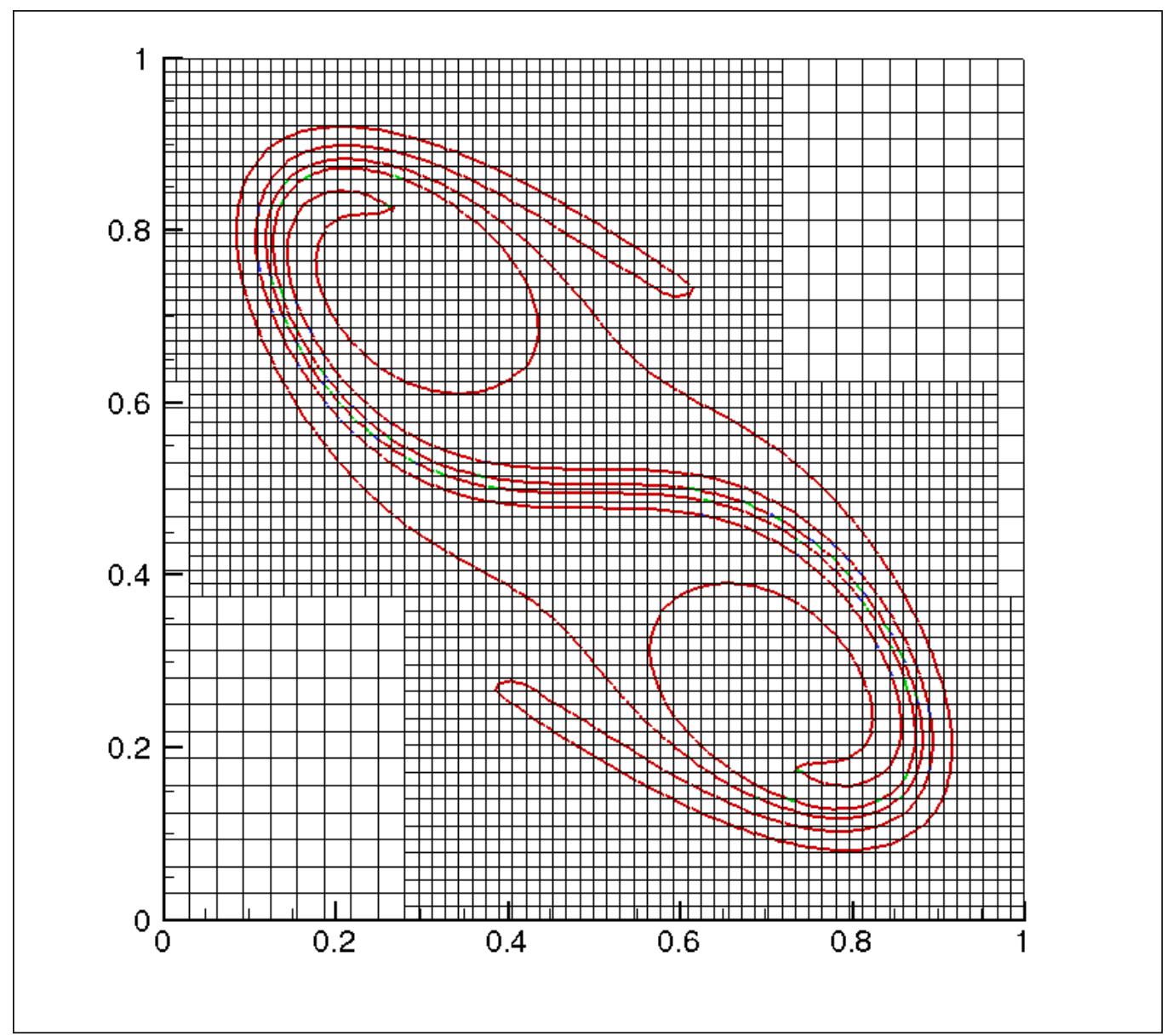

Figure 27: The S-shape flow at maximum deformation with a base grid mesh resolution of $32^{2}$, one level of AMR, and filament capturing enabled. Blue lines indicate the introduction of a fictitious Material $1^{\prime}$ to capture a filament of Material 2. A green line indicates the introduction of a fictitious Material $2^{\prime}$ to capture a filament of Material 1 . Note that filaments of both material types are formed. 


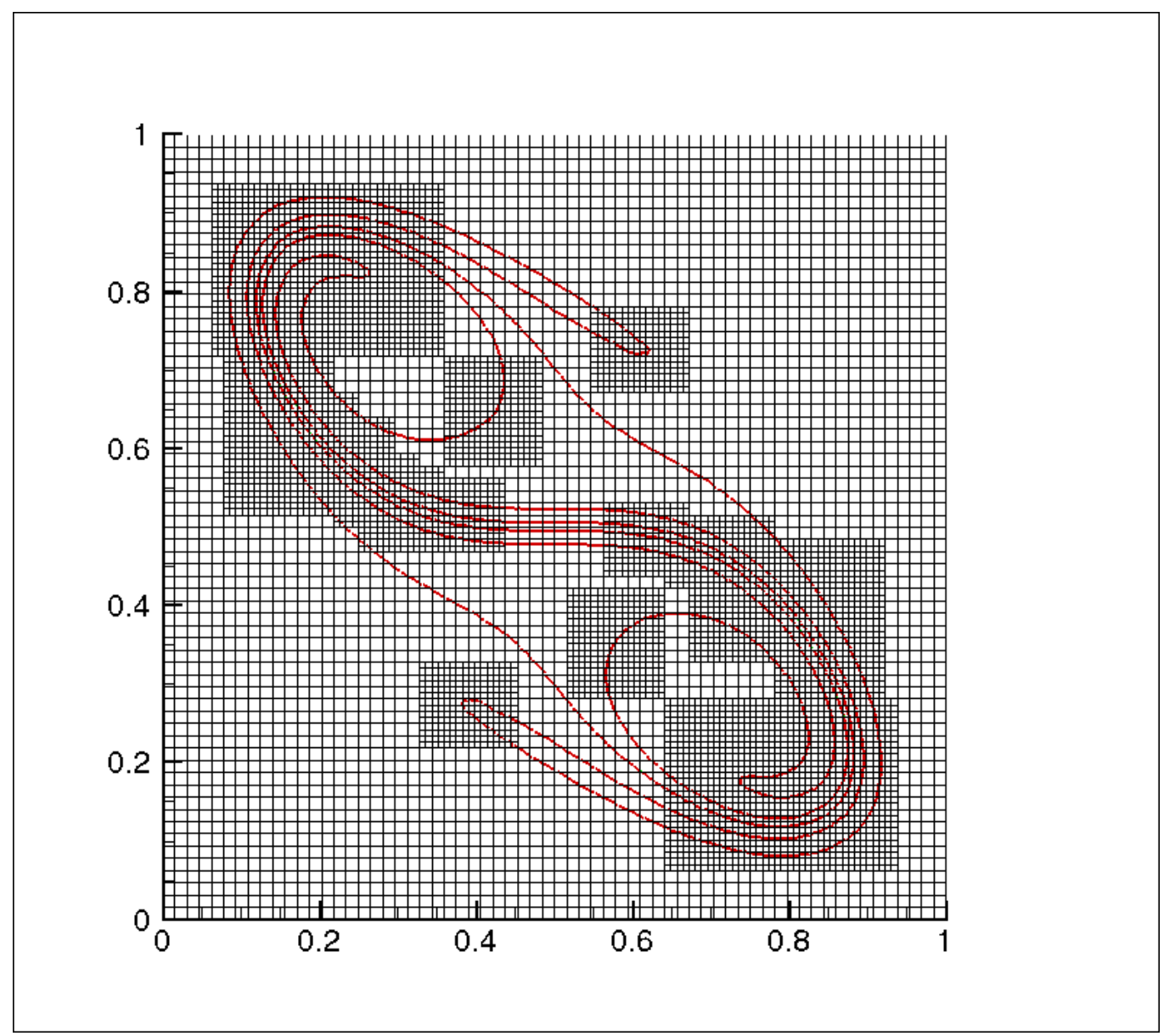

Figure 28: The S-shape flow at maximum deformation with a base grid mesh resolution of $32^{2}$, two levels of AMR, and filament capturing enabled. Blue lines indicate the introduction of a fictitious Material $1^{\prime}$ to capture a filament of Material 2. A green line indicates the introduction of a fictitious Material $2^{\prime}$ to capture a filament of Material 1. 


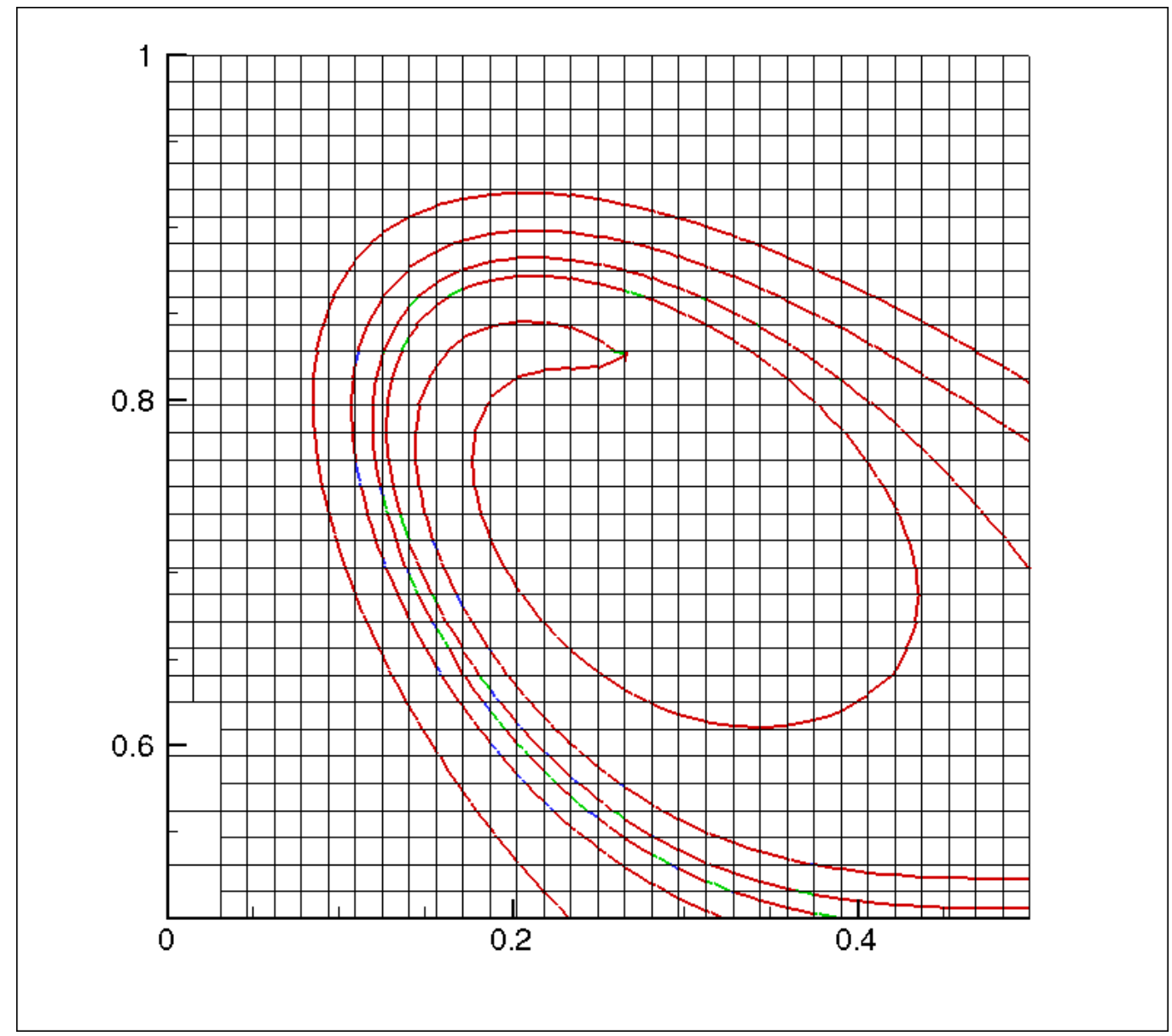

Figure 29: The S-shape interface from Fig. 27 (1 level of AMR), zoomed in near the vortex center to show captured filaments of each material type. 


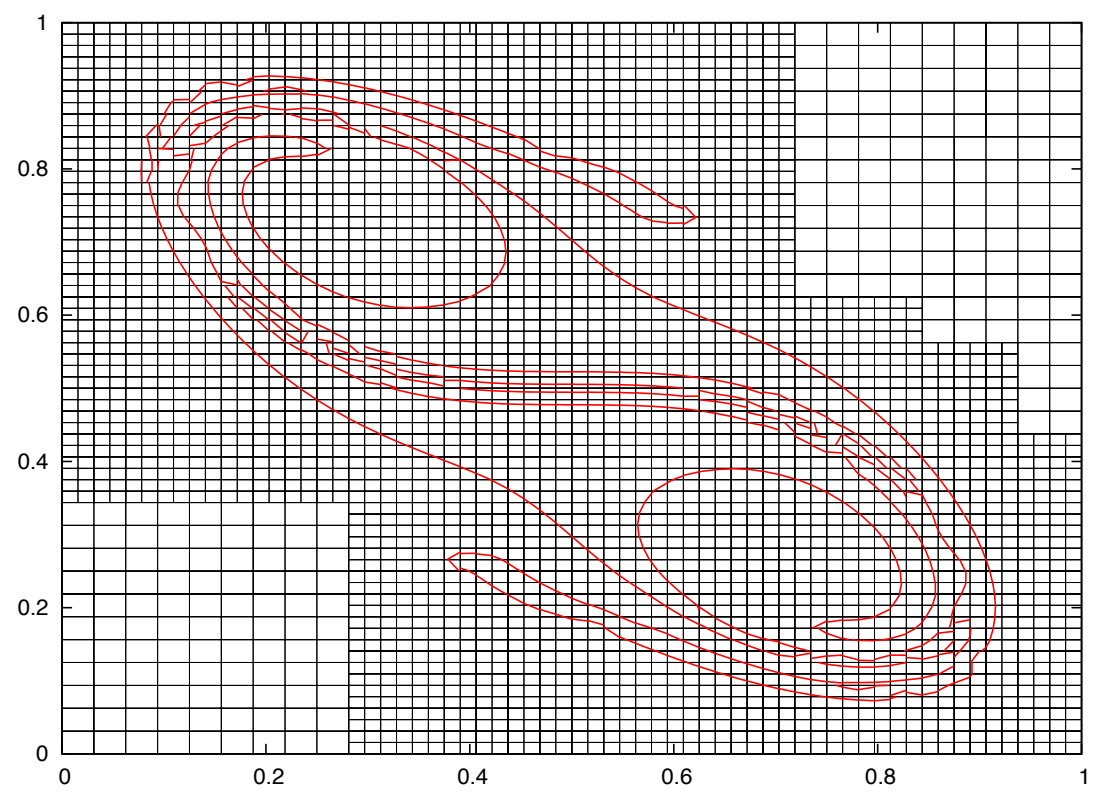

Figure 30: The S-shape flow at maximum deformation with a base grid mesh resolution of $32^{2}, 1$ level of AMR, and using the MOF method with filament capturing disabled. Note the break-up of thin, under-resolved structures due to deformation. 


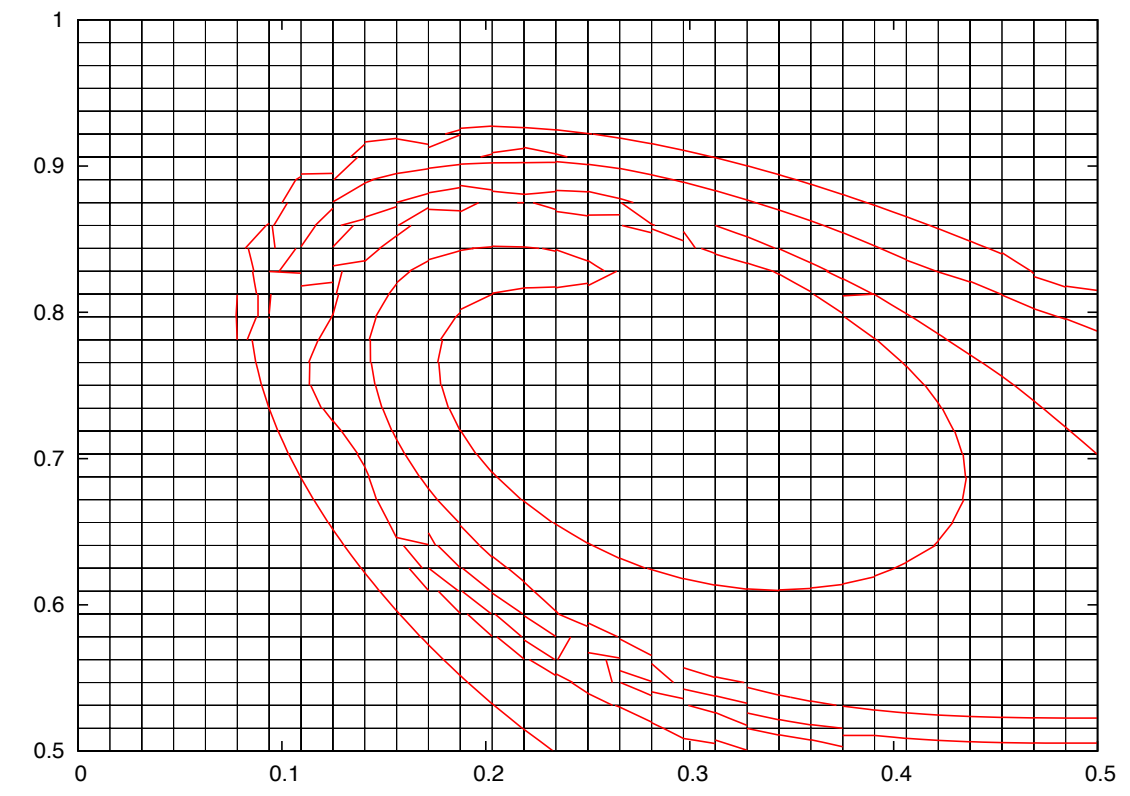

Figure 31: The S-shape interface from Fig. 30, zoomed in near the vortex center to show numerical break-up. 


\subsection{Pulsating Membrane}

We introduce a new test problem to demonstrate the unique functionality of our method. A thin, circular shell with outer-radius $r_{0}=1.0$ and inner-radius $r_{1}=1.0-\epsilon$ is initially located at the center of the $[-2,2]^{2}$ domain. A nonlinear, divergence-free velocity field causes expansion in the horizontal direction and compression in the vertical direction, until time $T / 4$, at which point the flow reverses and the material returns to its initial configuration at time $T / 2$. The shell is then compressed in the horizontal direction until time $3 T / 4$, and then returns back to its original shape. Figures 32-36 show the membrane interface, which is under-resolved by the mesh, undergoing deformation by the flow for four periods. Note that there is no break-up of the interface.

The velocity field is given by (6.6). Here, period time $T=2.0$, and the membrane has thickness 0.02 . Initial mesh resolution is $32^{2}$.

$$
\boldsymbol{u}(x, y, t)=\left[\begin{array}{c}
\sin (\pi x / 2) \cos (\pi y / 2) \cos (2 \pi t / T) \\
-\cos (\pi x / 2) \sin (\pi y / 2) \cos (2 \pi t / T)
\end{array}\right]
$$




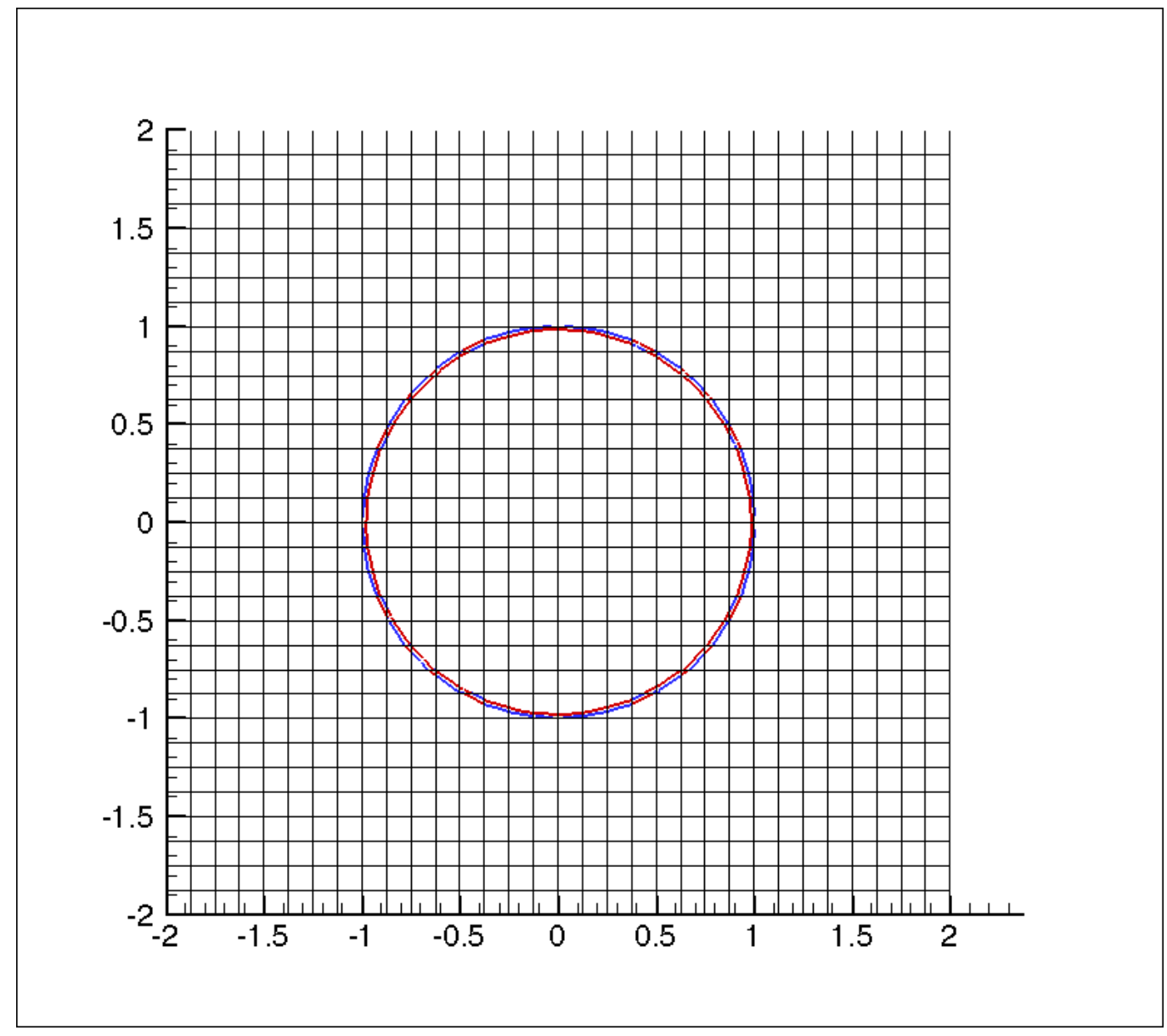

Figure 32: Initial interface configuration of the pulsating membrane. 


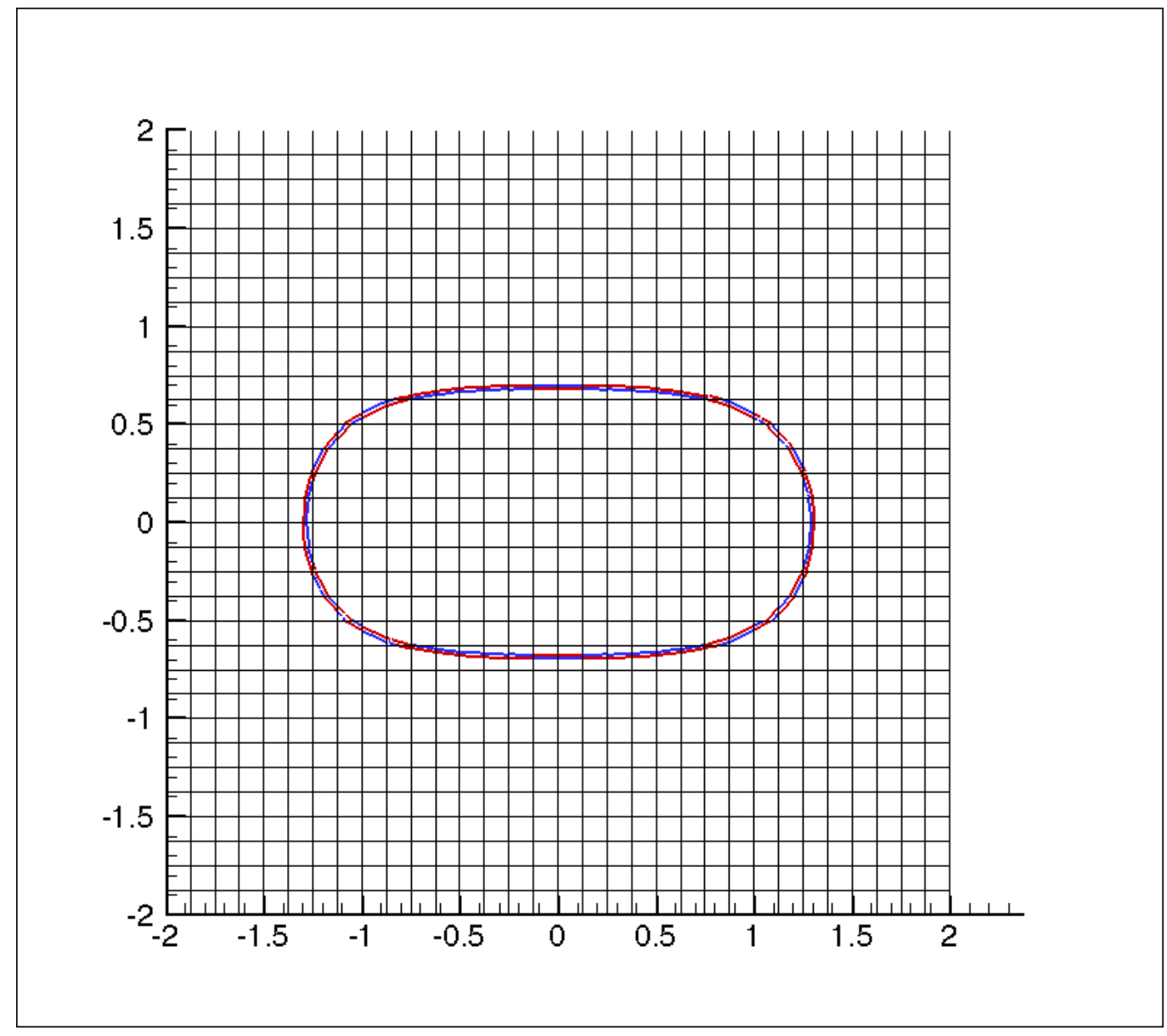

Figure 33: Pulsating membrane at time $t=0.5$. 


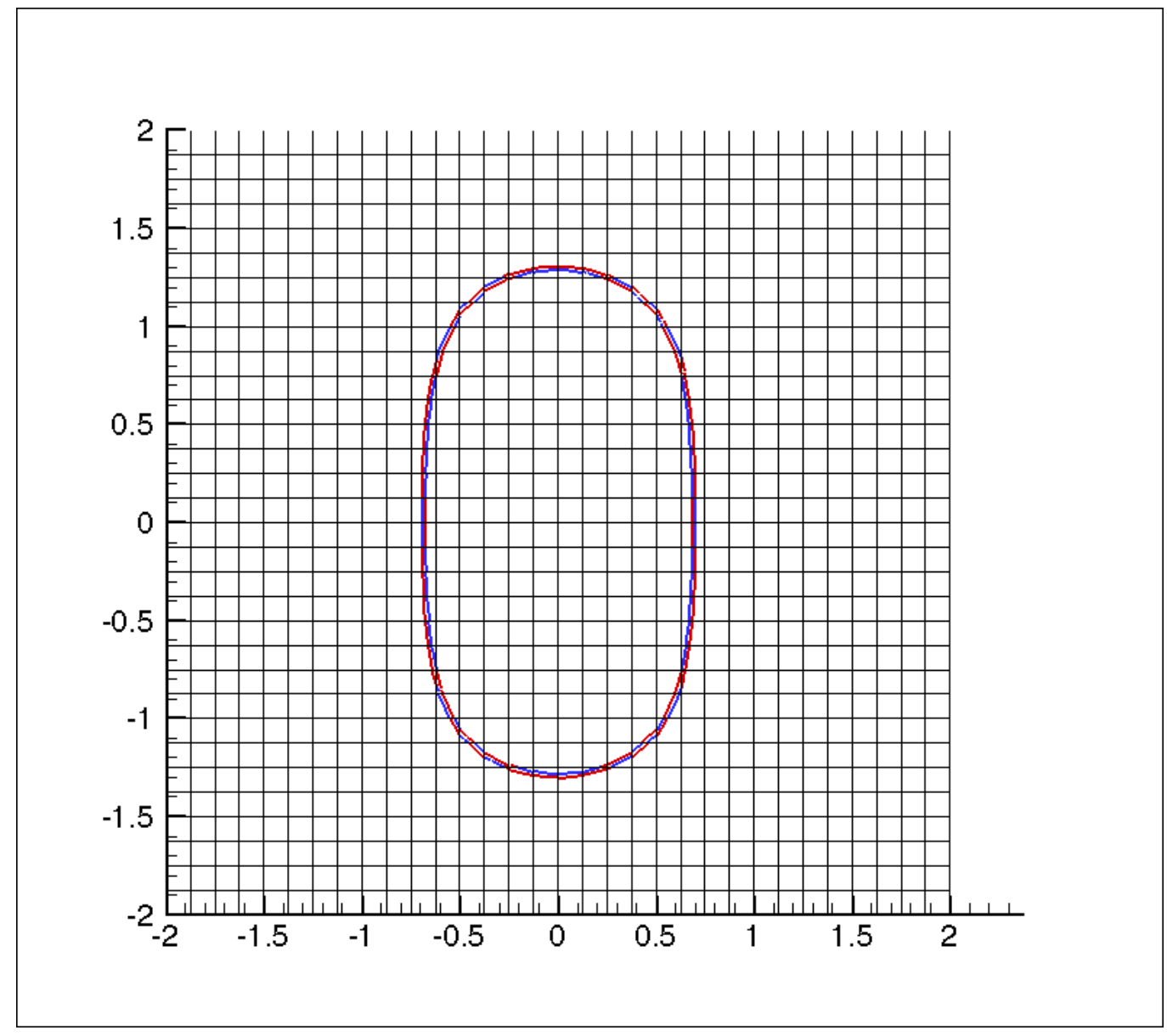

Figure 34: Pulsating membrane at time $t=1.5$. 


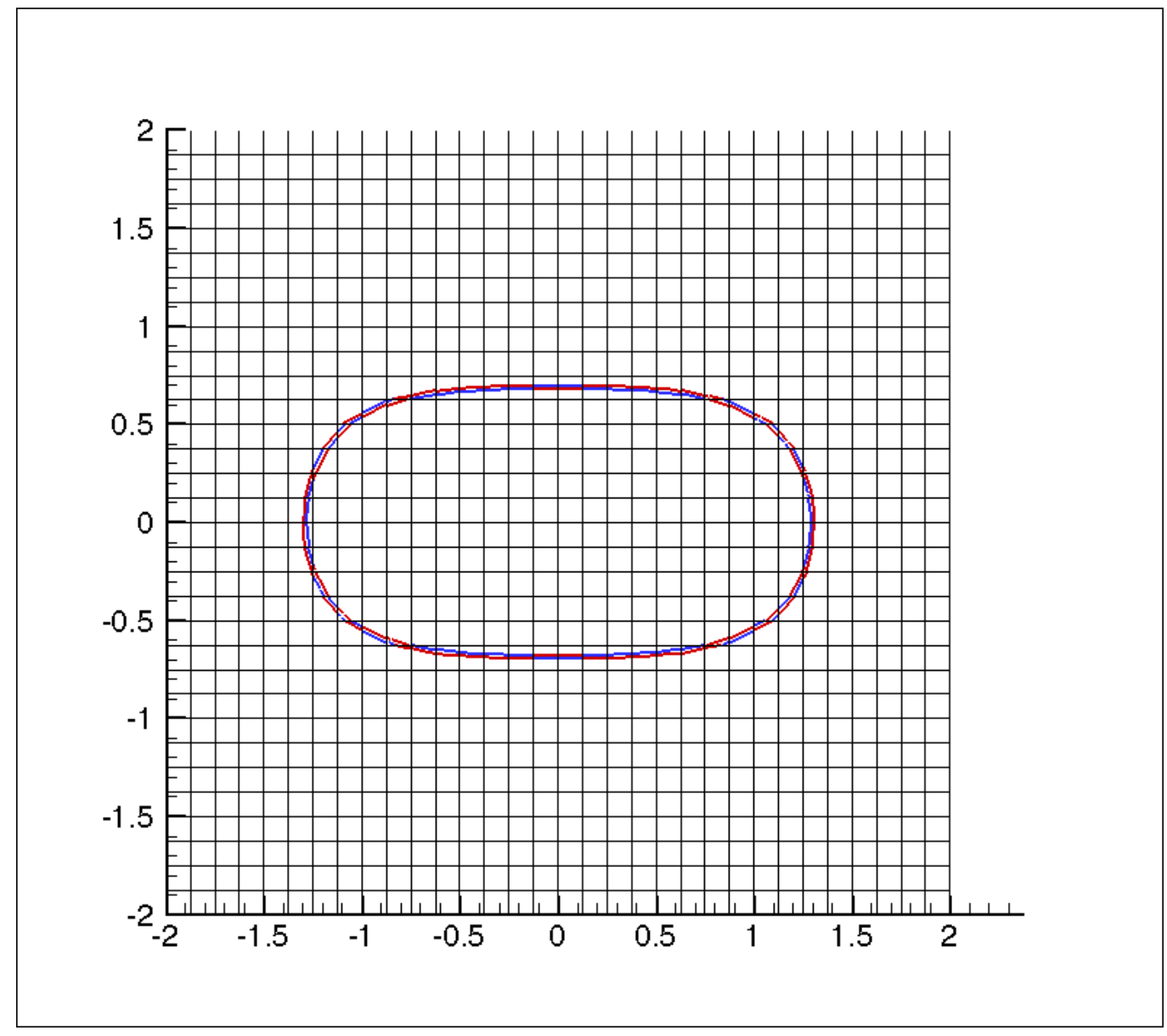

Figure 35: Pulsating membrane at time $t=4.5$. 


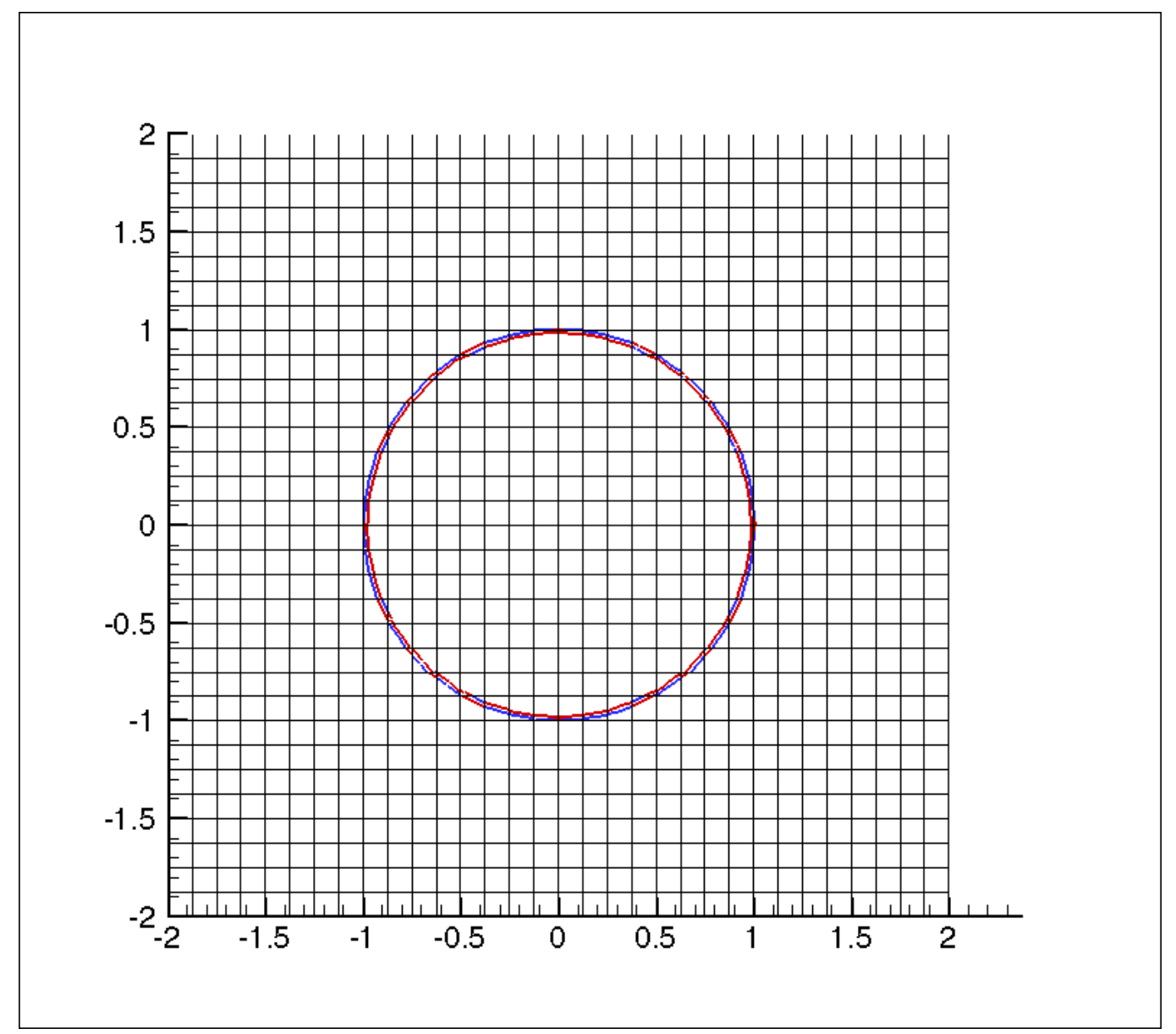

Figure 36: Pulsating membrane at the final time $t=8$. The thin interface has not experienced break-up, despite deformation due to the flow. 


\section{Conclusions}

A new method for capturing thin interfacial structures, under-resolved by the mesh, has been developed in the context of the Moment-ofFluid method. The method detects disconnected regions of material in a computational cell's advective preimage via a conglomeration algorithm. If a filament is detected in the advective preimage, a fictitious material is introduced, and the interface reconstruction problem becomes a problem of three materials separated by two interfaces rather than two materials separated by a single interface. This technique is seen to preserve thin structures under deformational flows, preventing spurious breakup when the material configuration is not well-resolved by the mesh. Performance of the Moment-of-Fluid method with filament capturing is compared to the standard Moment-of-Fluid method, with favorable results. In all cases, the use of filament capturing at a given level of mesh refinement produces results that are more accurate than using the standard Moment-of-Fluid method with an additional level of mesh refinement.

The procedure for Adaptive Mesh Refinement is developed in the context of the filament capturing method. The conglomeration procedure is used in the synchronization and error routines to allow for the introduction of mesh refinement near the tip of a filament or when a large reconstruction error is detected, but does not introduce refinement when the interface is accurately captured by a filament.

Future work includes the application of filament capturing in physics simulations, to include simulating skirted bubbles, deforming membranes, and viscous/visco-elastic fluid flow. The conglomeration procedure and filament capturing can be applied to merging liquid/liquid droplets to improve surface tension calculation as the two droplets enter the same cell. When a fictitious material is introduced, the materials' density, momentum, and energy will be initialized as well so that material states on either side of a filament are automatically kept separate.

Our conglomeration algorithm was implemented for a rectangular mesh, but the conglomeration procedure is independent of mesh structure, meaning it can be implemented in conjunction with a Multimaterial Moment-of-Fluid code to capture filaments on unstructured meshes. Finally, the method can be extended to three dimensions by altering the conglomeration procedure to detect adjacency between tetrahedra. The introduction of a fictitious material would then allow 
for capturing filamentary "sheet" structures in three dimensions.

\section{References}

[1] H. Ahn and M. Shashkov. Multi-material interface reconstruction on generalized polyhedral meshes. J. Comput. Phys., 226(2):2096-2132, 2007.

[2] H. Ahn and M. Shashkov. Adaptive moment-of-fluid method. J. Comput. Phys., 228(8):2792-2821, 2009.

[3] J. C. Anderson, C. Garth, M. A. Duchaineau, and K. I. Joy. Smooth, volume-accurate material interface reconstruction. IEEE Transactions on Visualization and Computer Graphics, 16(5):802-814, 2010.

[4] G. Cerne, S. Petelin, and I. Tiselj. Coupling of the interface tracking and the two-fluid models for the simulation of incompressible two-phase flow. J. Comput. Phys., 171:776-804, 2001.

[5] V. Dyadechko and M. Shashkov. Reconstruction of multi-material interfaces from moment data. J. Comput. Phys., 227:5361-5384, 2008 .

[6] D. Enright, R. Fedkiw, J. Ferziger, and I. Mitchell. A hybrid particle level set method for improved interface capturing. $J$. Comp. Phys., 183(1):83-116, 2002.

[7] C. Fochesato, R. Loubère, R. Motte, and J. Ovadia. Adaptive subdivision piecewise linear interface calculation (asplic) for $2 \mathrm{~d}$ multi-material hydrodynamic simulation codes. Int. J. Num. Meth. Fluids (early view), 2014.

[8] M. M. Francois, R. B. Lowrie, and E. D. Dendy. A material interface transition algorithm for multiphase flow. In Proceedings of the FEDSM 08, ASME Fluids Engineering Division, Jacksonville, FL, USA, August 10-14 2008.

[9] R. Garimella, V. Dyadechko, B. Swartz, and M. Shashkov. Interface reconstruction in multi-fluid, multiphase flow simulations. In Proceedings of the 14th International Meshing Roundtable, San Diego, CA, USA, September 11-14 2005.

[10] M. Jemison, E. Loch, M. Sussman, M. Shashkov, M. Arienti, M. Ohta, and Y. Wang. A coupled level set-moment of fluid 
method for incompressible two-phase flows. J. Sci. Comput., 54(2-3):454-491, Feb. 2013.

[11] J.-C. Nave, R. R. Rosales, and B. Seibold. A gradient-augmented level set method with an optimally local, coherent advection scheme. J. Comput. Phys., 229:3802-3827, 2010.

[12] R. Nourgaliev and T. Theofanous. High-fidelity interface tracking in compressible flows: Unlimited anchored adaptive level set. Journal of Computational Physics, 224:836-866, 2007.

[13] R. Nourgaliev, S. Wiri, N. Dinh, , and T. Theofanous. On improving mass conservation of level set by reducing spatial discretization errors. Int. J. Multiphase Flow, 31:1329-1336, 2005.

[14] D. Peng, B. Merriman, S. Osher, H. Zhao, and M. Kang. A pde based fast local level set method. J. Comp. Phys., 155(2):410 438, Nov 1999.

[15] M. Raessi, J. Mostaghimi, and M. Bussmann. Advecting normal vectors: A new method for calculating interface normals and curvatures when modeling two-phase flows. J. Comput. Phys., 226:774-797, 2007.

[16] Y. Renardy and M. Renardy. PROST: A Parabolic Reconstruction of Surface Tension for the Volume-of-Fluid Method. Journal of Computational Physics, 183(2):400-421, Dec. 2002.

[17] S. Shin and D. Juric. Modeling three-dimensional multiphase flow using a level contour reconstruction method for front tracking without connectivity. J. Comp. Phys., 180(2):427-470, 2002.

[18] C. Sijoy and S. Chaturvedi. Volume-of-fluid algorithm with different modified dynamic material ordering methods and their comparisons. Journal of Computational Physics, 229(10):3848 - 3863, 2010 .

[19] S.Leung and H.Zhao. A grid based particle method for moving interface problems. J. Comp. Phys., 228:2993-3204, 2009.

[20] S.Leung, J. Lowengrub, and H.Zhao. A grid based particle method for high order geometrical motions and local inextensible flows. J. Comp. Phys., 230(7):2540-2561, 2011.

[21] M. Sussman, A. Almgren, J. Bell, P. Colella, L. Howell, and M. Welcome. An adaptive level set approach for incompressible two-phase flows. J. Comput. Phys., 148:81-124, 1999. 
[22] M. Sussman and M. Hussaini. A discontinuous spectral element method for the level set equation. J. Scientific Computing, 19:479-500, 2003.

[23] M. Sussman, P. Smereka, and S. Osher. A level set approach for computing solutions to incompressible two-phase flow. J. Comput. Phys., 114:146-159, 1994.

[24] S. O. Unverdi and G. Tryggvason. A front-tracking method for viscous, incompressible, multi-fluid flows. J. Comput. Phys., 100:25-37, 1992.

[25] Q. Zhang and P. L.-F. Liu. A new interface tracking method: The polygonal area mapping method. Journal of Computational Physics, 227(8):4063 - 4088, 2008. 